Molecules 2007, 12, 25-42

\title{
molecules
}

ISSN 1420-3049

http://www.mdpi.org

\author{
Full Paper
}

\section{An Efficient Synthesis and Reactions of Novel Indolyl- pyridazinone Derivatives with Expected Biological Activity}

\author{
Samar A. Abubshait \\ Chemistry Department, Girls College of Science, P.O. Box 838, Dammam-31113, Saudi Arabia, Tel: \\ (+966) 504988846, Fax: (+966) 38681088; E-mail: abu_sa_005@yahoo.com
}

Received: 29 October 2006; in revised form: 9 December 2006 / Accepted: 18 December 2006 / Published: 10 January 2007

\begin{abstract}
Reaction of 4-anthracen-9-yl-4-oxo-but-2-enoic acid (1) with indole gave the corresponding butanoic acid 2. Cyclocondensation of 2 with hydrazine hydrate, phenyl hydrazine, semicarbazide and thiosemicarbazide gave the pyridazinone derivatives 3ad. Reaction of $3 \mathbf{a}$ with $\mathrm{POCl}_{3}$ for 30 min gave the chloropyridazine derivative $\mathbf{4 a}$, which was used to prepare the corresponding carbohydrate hydrazone derivatives 5a-d. Reaction of chloropyridazine 4a with some aliphatic or aromatic amines and anthranilic acid gave 6a-f and 7 , respectively. When the reaction of the pyridazinone derivative 3a with $\mathrm{POCl}_{3}$ was carried out for $3 \mathrm{hr}$ an unexpected product $\mathbf{4 b}$ was obtained. The structure of $\mathbf{4 b}$ was confirmed by its reaction with hydrazine hydrate to give hydrazopyridazine derivative 9, which reacted in turn with acetyl acetone to afford 10. Reaction of $\mathbf{4 b}$ with methylamine gave $\mathbf{1 1}$, which reacted with methyl iodide to give the trimethylammonium iodide derivative 12. The pyridazinone $3 \mathbf{a}$ also reacted with benzene- or 4-toluenesulphonyl chloride to give 13a-b and with aliphatic or aromatic aldehydes to give 14a-g. All proposed structures were supported by IR, ${ }^{1} \mathrm{H}-\mathrm{NMR},{ }^{13} \mathrm{C}$ NMR, and MS spectroscopic data. Some of the new products showed antibacterial activity.
\end{abstract}

Keywords: Substituted pyridazinones, chloropyridazine, anthracene, indole, antibacterial activity. 


\section{Introduction}

In recent years a substantial number of 6-aryl-3- $(2 H)$-pyridazinones have been reported to possess antimicrobial [1, 2], potent analgesic [3], anti-inflammatory [3-7], antifeedant [8], herbicidal [9], antihypertensive [10-12] and antiplatelet activities [13-15], anticancer effects [16] and other anticipated biological [17] and pharmacological properties [18, 19]. In particular, a large number of indolylpyridazinone derivatives are well known as antimicrobial agents [1, 20], intermediates for drugs and agrochemicals [21, 22], antiphlogistics [23], antipyretics [24], inflammation inhibitors [25], blood platelet aggregation inhibitors, cardiovascular and antihypertensive agents [26]. As part of our program aimed at utilizing $\beta$-aroylpropionic acid derivatives containing the indole moiety as starting materials for the synthesis of pyridazine and pyridazinone derivatives, these reports of interesting biological activities prompted us to synthesize a new series of 6-anthracenepyridazinones containing indolyl moieties through the nucleophilic addition of indole to 6-anthracene-4-oxo-2-butenoic acid, followed by cyclocondensation of the resulting adduct to give the corresponding dihydropyridazinone and to screen some of these new compounds for antibacterial activity.

\section{Results and Discussion}

4-Anthracen-9-yl-4-oxo-but-2-enoic acid (1) was prepared following a reported procedure [1, 27]. Reaction of 1 with indole in dry benzene gave 4-anthracen-9-yl-2-(1H-indol-3-yl)-4-oxo-butyric acid (2). Cyclocondensation of 2 with hydrazine hydrate, phenyl hydrazine, semicarbazide and thiosemicarbazide in dry benzene [28-31] gave 6-anthracen-9-yl-4-(1H-indol-3-yl)-4,5-dihydro- $2 \mathrm{H}$ pyridazin-3-one, 6-anthracen-9-yl-4-(1H-indol-3-yl)-2-phenyl-4,5-dihydro-2H-pyridazin-3-one, 3anthracen-9-yl-5-(1H-indol-3-yl)-6-oxo-5,6-dihydro-4H-pyridazine-1-carboxylic acid amide and 3anthracen-9-yl-5-(1H-indol-3-yl)-6-oxo-5,6-dihydro-4H-pyridazine-1-carbothioic acid amides 3a-d, respectively (Scheme 1). Physical properties, mass spectral data and elemental analyses for the synthesized compounds $\mathbf{1 - 3 d}$ are given in Table 1.

\section{Scheme 1.}

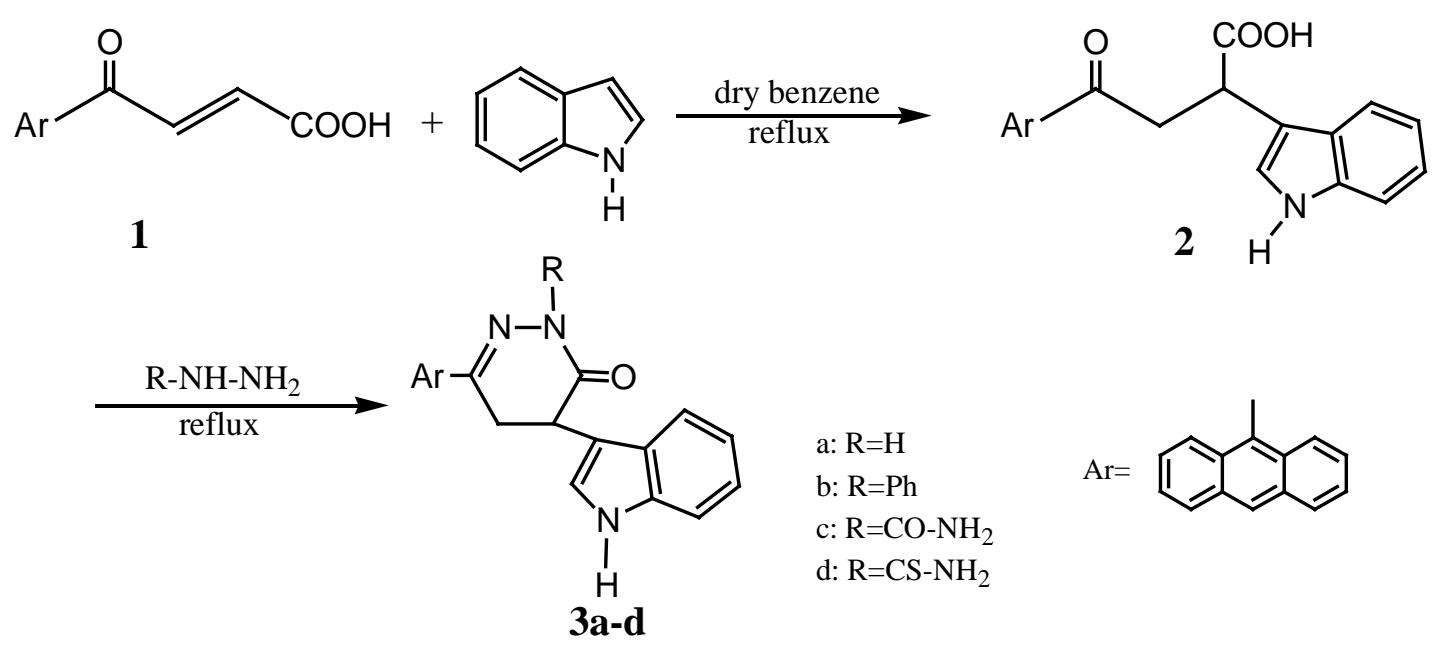


Table 1. Physical properties, mass spectral data and elemental analyses for compounds 1-3.

\begin{tabular}{|c|c|c|c|c|c|c|c|}
\hline \multirow{2}{*}{$\begin{array}{c}\text { Compound } \\
\text { No }\end{array}$} & \multirow{2}{*}{$\begin{array}{l}\text { M.P. }\left({ }^{\circ} \mathrm{C}\right) \\
\text { Cryst. solvent }\end{array}$} & \multirow{2}{*}{$\begin{array}{l}\text { Mol. Formula } \\
\text { Mol. weight }\end{array}$} & \multirow{2}{*}{$\begin{array}{l}\text { M.W. } \\
\text { from MS }\end{array}$} & \multicolumn{4}{|c|}{ Analysis \% Calc./ Found } \\
\hline & & & & C & $\mathbf{H}$ & $\mathbf{N}$ & $\mathbf{S}$ \\
\hline \multirow[t]{2}{*}{1} & $215-217$ & $\mathrm{C}_{18} \mathrm{H}_{12} \mathrm{O}_{3}$ & 276.08 & 78.25 & 4.38 & ----- & ----- \\
\hline & Ethanol & 276.29 & & 78.20 & 4.35 & & \\
\hline \multirow[t]{2}{*}{2} & 223 & $\mathrm{C}_{26} \mathrm{H}_{19} \mathrm{NO}_{3}$ & 393.14 & 79.37 & 4.87 & 3.56 & ----- \\
\hline & Benzene & 393.43 & & 79.35 & 4.82 & 3.53 & \\
\hline \multirow[t]{2}{*}{ 3a } & 122 & $\mathrm{C}_{26} \mathrm{H}_{19} \mathrm{~N}_{3} \mathrm{O}$ & 389.15 & 80.18 & 4.92 & 10.79 & ----- \\
\hline & Benzene & 389.45 & & 80.20 & 4.72 & 10.59 & \\
\hline \multirow[t]{2}{*}{$3 \mathbf{b}$} & 270 & $\mathrm{C}_{32} \mathrm{H}_{23} \mathrm{~N}_{3} \mathrm{O}$ & 465.18 & 82.56 & 4.98 & 9.03 & ----- \\
\hline & Benzene & 465.54 & & 82.58 & 4.94 & 9.06 & \\
\hline \multirow[t]{2}{*}{ 3c } & 145 & $\mathrm{C}_{27} \mathrm{H}_{20} \mathrm{~N}_{4} \mathrm{O}_{2}$ & 432.16 & 74.98 & 4.66 & 12.96 & ----- \\
\hline & Benzene & 432.47 & & 75.01 & 4.70 & 13.00 & \\
\hline \multirow[t]{2}{*}{ 3d } & 160 & $\mathrm{C}_{27} \mathrm{H}_{20} \mathrm{~N}_{4} \mathrm{OS}$ & 448.14 & 72.30 & 4.49 & 12.49 & 7.15 \\
\hline & Benzene & 448.54 & & 72.37 & 4.53 & 12.52 & 7.20 \\
\hline
\end{tabular}

Reaction of pyridazinone 3a with $\mathrm{POCl}_{3}$ for 30 min gave the chloropyridazine derivative 4a [32], which reacted with carbohydrate hydrazones of ribose, glucose, galactose and lactose in ethanol to give hydrazonopyradazine derivatives 5a-d [6, 12]. Mixing chloropyridazine 4a with aliphatic or aromatic amines, namely methylamine, ethylamine, aniline, sulphanilinic acid, $\alpha$-naphthylamine or diphenylamine in dry benzene gave pyridazine derivatives $\mathbf{6 a - f}$ [7, 12, 33]. In addition, $\mathbf{4 a}$ reacted with anthranilic acid in dry benzene to give 7 (Scheme 2) [1, 30, 32]. Physical properties, mass spectral data and elemental analyses for all newly synthesized compounds $\mathbf{4 a - 7}$ are listed in Table 2.

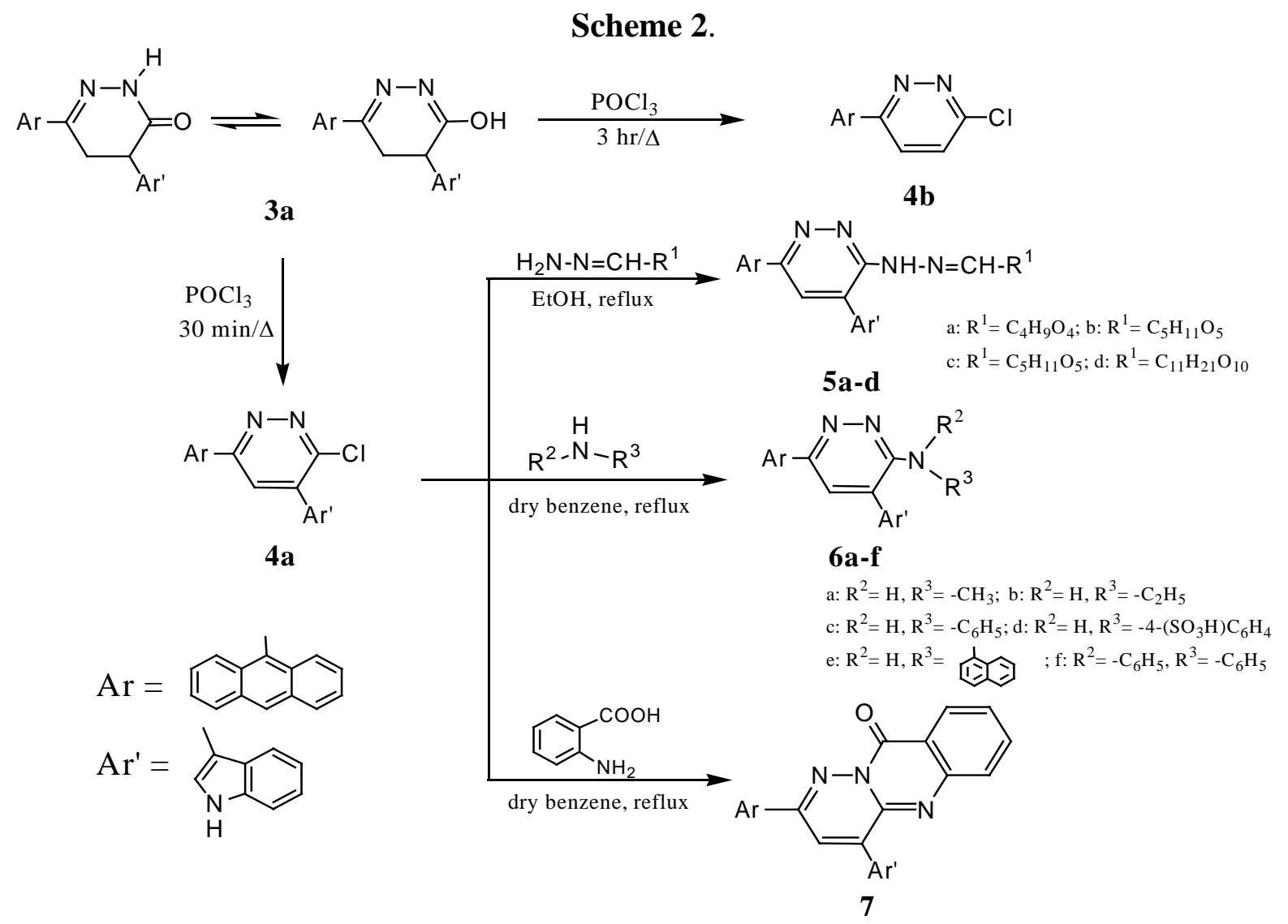


Table 2. Physical properties, mass spectral data and elemental analyses for compounds 4-7.

\begin{tabular}{|c|c|c|c|c|c|c|c|}
\hline \multirow{2}{*}{$\begin{array}{c}\text { Compound } \\
\text { No }\end{array}$} & \multirow{2}{*}{$\begin{array}{l}\text { M.P. }\left({ }^{\circ} \mathrm{C}\right) \\
\text { Cryst. solvent }\end{array}$} & \multirow{2}{*}{$\begin{array}{l}\text { Mol. Formula } \\
\text { Mol. Weight }\end{array}$} & \multirow{2}{*}{$\begin{array}{l}\text { M.W. } \\
\text { from MS }\end{array}$} & \multicolumn{4}{|c|}{ Analysis \% Calc./ Found } \\
\hline & & & & C & $\mathbf{H}$ & $\mathbf{N}$ & $\mathbf{S}$ \\
\hline \multirow[t]{2}{*}{$4 a$} & 200 & $\mathrm{C}_{26} \mathrm{H}_{16} \mathrm{ClN}_{3}$ & 405.10 & 76.94 & 3.97 & 10.35 & ----- \\
\hline & Benzene & 405.88 & & 76.99 & 3.94 & 10.33 & \\
\hline \multirow[t]{2}{*}{$4 b$} & 152 & $\mathrm{C}_{18} \mathrm{H}_{11} \mathrm{ClN}_{2}$ & 290.06 & 74.36 & 3.81 & 9.64 & ---- \\
\hline & Benzene & 290.75 & & 74.40 & 3.85 & 9.66 & \\
\hline \multirow[t]{2}{*}{$5 a$} & 360 & $\mathrm{C}_{31} \mathrm{H}_{27} \mathrm{~N}_{5} \mathrm{O}_{4}$ & 533.56 & 69.78 & 5.10 & 13.13 & ----- \\
\hline & Ethanol & 533.58 & & 69.82 & 5.15 & 13.17 & \\
\hline \multirow[t]{2}{*}{$5 b$} & 315 & $\mathrm{C}_{32} \mathrm{H}_{29} \mathrm{~N}_{5} \mathrm{O}_{5}$ & 563.60 & 68.19 & 5.19 & 12.43 & ----- \\
\hline & Ethanol & 563.60 & & 68.23 & 5.23 & 12.40 & \\
\hline \multirow[t]{2}{*}{$5 c$} & 348 & $\mathrm{C}_{32} \mathrm{H}_{29} \mathrm{~N}_{5} \mathrm{O}_{5}$ & 563.60 & 68.19 & 5.19 & 12.43 & ----- \\
\hline & Ethanol & 563.60 & & 68.14 & 5.23 & 12.44 & \\
\hline \multirow[t]{2}{*}{$5 d$} & $138-140$ & $\mathrm{C}_{38} \mathrm{H}_{39} \mathrm{~N}_{5} \mathrm{O}_{10}$ & 725.24 & 62.89 & 5.42 & 9.65 & ----- \\
\hline & Ethanol & 725.74 & & 62.93 & 5.46 & 9,63 & \\
\hline \multirow[t]{2}{*}{$6 a$} & 147 & $\mathrm{C}_{27} \mathrm{H}_{20} \mathrm{~N}_{4}$ & 400.17 & 80.98 & 5.03 & 13.99 & ----- \\
\hline & Benzene & 400.47 & & 80.95 & 5.08 & 14.02 & \\
\hline \multirow[t]{2}{*}{$6 b$} & 210 & $\mathrm{C}_{28} \mathrm{H}_{22} \mathrm{~N}_{4}$ & 414.18 & 81.13 & 5.35 & 13.52 & ----- \\
\hline & Benzene & 414.50 & & 81.20 & 5.40 & 13.56 & \\
\hline \multirow[t]{2}{*}{$6 c$} & 156 & $\mathrm{C}_{32} \mathrm{H}_{22} \mathrm{~N}_{4}$ & 462.54 & 83.09 & 4.79 & 12.11 & ----- \\
\hline & Benzene & 462.54 & & 83.12 & 4.74 & 12.09 & \\
\hline \multirow[t]{2}{*}{ 6d } & 286 & $\mathrm{C}_{32} \mathrm{H}_{22} \mathrm{~N}_{4} \mathrm{O}_{3} \mathrm{~S}$ & 542.14 & 70.83 & 4.09 & 10.33 & 5.91 \\
\hline & Benzene & 542.61 & & 70.79 & 4.00 & 10.00 & 5.94 \\
\hline \multirow[t]{2}{*}{$6 e$} & 199 & $\mathrm{C}_{36} \mathrm{H}_{24} \mathrm{~N}_{4}$ & 512.22 & 84.35 & 4.72 & 10.93 & ----- \\
\hline & Benzene & 512.60 & & 84.40 & 4.76 & 10.95 & \\
\hline \multirow[t]{2}{*}{$6 f$} & 236 & $\mathrm{C}_{38} \mathrm{H}_{26} \mathrm{~N}_{4}$ & 538.22 & 84.73 & 4.87 & 10.40 & ----- \\
\hline & Benzene & 538.64 & & 84.77 & 4.82 & 10.46 & \\
\hline \multirow[t]{2}{*}{7} & 130 & $\mathrm{C}_{33} \mathrm{H}_{20} \mathrm{~N}_{4} \mathrm{O}$ & 488.16 & 81.13 & 4.13 & 11.47 & ----- \\
\hline & Benzene & 488.54 & & 81.16 & 4.17 & 11.50 & \\
\hline
\end{tabular}

Surprisingly, when the reaction of 3a with $\mathrm{POCl}_{3}$ was carried out for $3 \mathrm{hr}$ an unexpected product, 6-anthracen-9-yl-6-chloropyridazine (4b), was obtained via dearylation and substitution of the hydroxyl group by chlorine [20] (Scheme 2). The structure of $\mathbf{4 b}$ was proven by the similarity of its melting point to that of an authentic sample which was independently prepared by the reaction of 4anthracen-9-yl-4-oxo-but-2-enoic acid (1) with hydrazine hydrate in dry benzene and treatment of the resulting pyridazinone $\mathbf{8}$ with $\mathrm{POCl}_{3}$ for $30 \mathrm{~min}$ to give $\mathbf{4 b}$ (Scheme 3) [1, 5, 7, 30, 31]. Reaction of chloropyridazine $\mathbf{4 b}$ with hydrazine hydrate in boiling benzene [34] gave the hydrazinopyridazine derivative 9, whose structure was inferred from its infrared spectrum. The structure of 9 was further confirmed by its reaction with acetyl acetone in boiling methanol that gave 3-anthracen-9-yl-6-(3,5dimethylpyrazol-1-yl) pyridazine (10) [5, 6, 14, 32]. As a point of interest, it was observed that reaction of $\mathbf{4 b}$ with methylamine at $140{ }^{\circ} \mathrm{C}$ afforded $\mathbf{1 1}$. On the other hand, when compound 11 was reacted with excess $\mathrm{CH}_{3} \mathrm{I}$ in methanol the quaternary ammonium iodide derivative $\mathbf{1 2}$ was formed (Scheme 3). Physical properties, mass spectral data and elemental analyses for all newly synthesized compounds 8-12 are given in Table 3. 
Scheme 3.

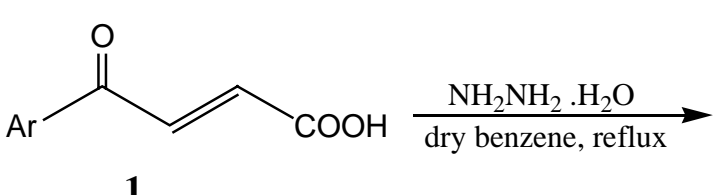

1<smiles>O=c1ccc(Br)n[nH]1</smiles><smiles>C=C=C</smiles>

8<smiles></smiles>

$4 \mathbf{b}$ 9

10<smiles>CNc1ccc(Br)nn1</smiles>

11<smiles>C[N+](C)(C)c1ccc(Br)nn1</smiles>

12

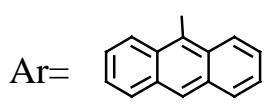

Table 3. Physical properties, mass spectral data and elemental analyses for compounds 8-12.

\begin{tabular}{|c|l|l|l|l|l|l|l|}
\hline \multirow{2}{*}{$\begin{array}{c}\text { Compound } \\
\text { No }\end{array}$} & M.P. $\left({ }^{\circ} \mathbf{C}\right)$ & Mol. Formula & M.W. & \multicolumn{3}{|c|}{ Analysis \% Calc./ Found } \\
\cline { 5 - 9 } & Cryst. solvent & Mol. Weight & from MS & C & H & N & S \\
\hline $\mathbf{8}$ & 165 & $\mathrm{C}_{18} \mathrm{H}_{12} \mathrm{~N}_{2} \mathrm{O}$ & 272.30 & 79.39 & 4.44 & 10.29 & ----- \\
& Benzene & 272.30 & & 79.42 & 4.40 & 10.32 & \\
$\mathbf{9}$ & 230 & $\mathrm{C}_{18} \mathrm{H}_{14} \mathrm{~N}_{4}$ & 286.12 & 75.50 & 4.93 & 19.57 & ---- \\
& Benzene & 286.33 & & 75.50 & 4.90 & 19.59 & \\
$\mathbf{1 0}$ & 210 & $\mathrm{C}_{23} \mathrm{H}_{18} \mathrm{~N}_{4}$ & 350.15 & 78.83 & 5.18 & 15.99 & ----- \\
$\mathbf{1 1}$ & Methanol & 350.42 & & 78.88 & 5.15 & 16.02 & \\
& 126 & $\mathrm{C}_{19} \mathrm{H}_{15} \mathrm{~N}_{3}$ & 285.12 & 79.98 & 5.30 & 14.73 & ---- \\
$\mathbf{1 2}$ & Methanol & 285.34 & & 80.02 & 5.28 & 14.77 & \\
& 150 & $\mathrm{C}_{21} \mathrm{H}_{20} \mathrm{~N}_{3} \mathrm{I}$ & 314.17 & 57.15 & 4.57 & 9.52 & ---- \\
& Methanol & 441.31 & & 57.10 & 4.52 & 9.58 & \\
\hline
\end{tabular}

The reaction of pyridazinone 3a with benzene/4-toluenesulfonyl chloride and anhydrous $\mathrm{K}_{2} \mathrm{CO}_{3}$ in dry acetone at reflux for $24 \mathrm{hr}$ gave 6-anthracen-9-yl-4-(1H-indol-3-yl)-2-(benzenesulfonyl or 4toluenesulfonyl)-4,5-dihydro-2H-pyridazin-3-ones 13a and 13b, respectively. Pyridazinone 3a also reacted with same aliphatic and aromatic aldehydes namely formaldehyde, acetaldehyde, benzaldehyde, 2-hydroxybenzaldehyde, 4-hydroxybenzaldehyde, 2-methoxybenzaldehyde, 4methoxybenzaldehyde, 2,4-dimethoxybenzaldehyde, 2-hydroxynapthaldehyde and furfuraldehyde to give pyridazinone derivatives 14a-j, respectively (Scheme 4). Physical properties, mass spectral data and elemental analyses for all new compounds 13a-14j are given in Table 4. 
Scheme 4.

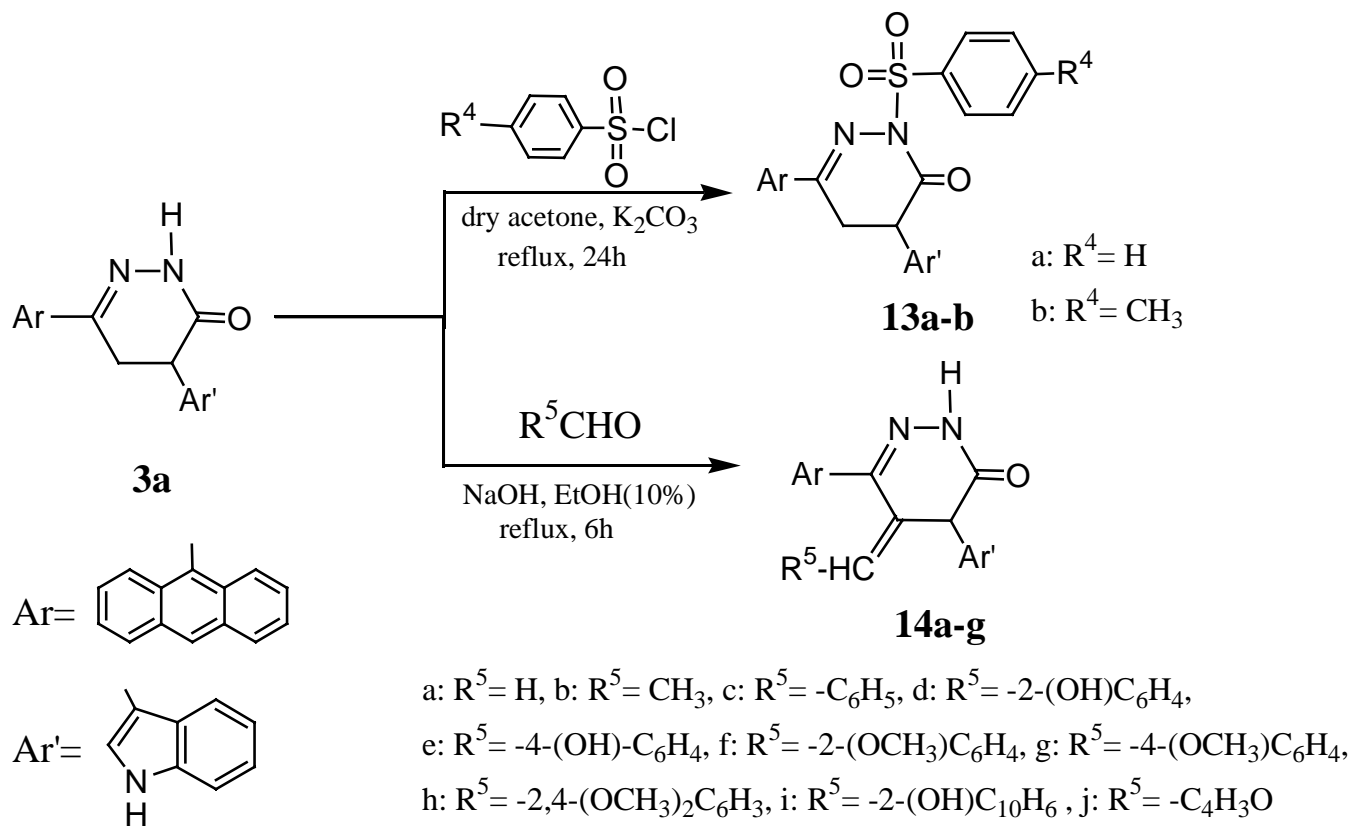

Table 4. Physical properties, mass spectral data and elemental analyses for compounds 13-14.

\begin{tabular}{|c|c|c|c|c|c|c|c|}
\hline \multirow{2}{*}{$\begin{array}{c}\text { Compound } \\
\text { No } \\
\end{array}$} & \multirow{2}{*}{$\begin{array}{l}\text { M.P. }\left({ }^{\circ} \mathrm{C}\right) \\
\text { Cryst. solvent }\end{array}$} & \multirow{2}{*}{$\begin{array}{l}\text { Mol. Formula } \\
\text { Mol. Weight }\end{array}$} & \multirow{2}{*}{$\begin{array}{l}\text { M.W. } \\
\text { from MS }\end{array}$} & \multicolumn{4}{|c|}{ Analysis \% Calc./ Found } \\
\hline & & & & C & $\mathbf{H}$ & $\mathbf{N}$ & S \\
\hline \multirow[t]{2}{*}{ 13a } & 154 & $\mathrm{C}_{32} \mathrm{H}_{23} \mathrm{~N}_{3} \mathrm{O}_{3} \mathrm{~S}$ & 529.15 & 72.57 & 4.38 & 7.93 & 6.05 \\
\hline & Benzene & 526.61 & & 72.50 & 4.41 & 7.90 & 6.00 \\
\hline \multirow[t]{2}{*}{$13 b$} & 177 & $\mathrm{C}_{33} \mathrm{H}_{25} \mathrm{~N}_{3} \mathrm{O}_{3} \mathrm{~S}$ & 543.16 & 72.91 & 4.64 & 7.73 & 5.90 \\
\hline & Benzene & 543.64 & & 72.95 & 4.68 & 7.77 & 5.93 \\
\hline \multirow[t]{2}{*}{$14 a$} & 260 & $\mathrm{C}_{27} \mathrm{H}_{19} \mathrm{~N}_{3} \mathrm{O}$ & 401.15 & 80.78 & 4.77 & 10.47 & ----- \\
\hline & Benzene & 401.46 & & 80.74 & 4.79 & 10.41 & \\
\hline \multirow[t]{2}{*}{$14 b$} & 288 & $\mathrm{C}_{28} \mathrm{H}_{21} \mathrm{~N}_{3} \mathrm{O}$ & 415.17 & 80.94 & 5.09 & 10.11 & ----- \\
\hline & Benzene & 415.49 & & 80.89 & 5.13 & 10.07 & \\
\hline \multirow[t]{2}{*}{$14 \mathrm{c}$} & 360 & $\mathrm{C}_{33} \mathrm{H}_{23} \mathrm{~N}_{3} \mathrm{O}$ & 477.18 & 83.00 & 4.85 & 8.80 & ----- \\
\hline & Benzene & 477.56 & & 83.03 & 4.88 & 8.85 & \\
\hline \multirow[t]{2}{*}{ 14d } & 350 & $\mathrm{C}_{33} \mathrm{H}_{23} \mathrm{~N}_{3} \mathrm{O}_{2}$ & 493.18 & 80.31 & 4.70 & 8.51 & ----- \\
\hline & Benzene & 493.55 & & 80.40 & 4.73 & 8.53 & \\
\hline \multirow[t]{2}{*}{$14 e$} & 264 & $\mathrm{C}_{33} \mathrm{H}_{23} \mathrm{~N}_{3} \mathrm{O}_{2}$ & 493.18 & 80.31 & 4.70 & 8.51 & ----- \\
\hline & Benzene & 493.55 & & 80.37 & 4.75 & 8.55 & \\
\hline \multirow[t]{2}{*}{$14 f$} & 196 & $\mathrm{C}_{34} \mathrm{H}_{25} \mathrm{~N}_{3} \mathrm{O}_{2}$ & 507.19 & 80.45 & 4.96 & 8.28 & ----- \\
\hline & Benzene & 507.58 & & 80.49 & 4.92 & 8.22 & \\
\hline \multirow[t]{2}{*}{$14 g$} & 202 & $\mathrm{C}_{34} \mathrm{H}_{25} \mathrm{~N}_{3} \mathrm{O}_{2}$ & 507.19 & 80.45 & 4.96 & 8.28 & ----- \\
\hline & Benzene & 507.58 & & 80.49 & 5.10 & 8.26 & \\
\hline \multirow[t]{2}{*}{$14 \mathrm{~h}$} & 94 & $\mathrm{C}_{35} \mathrm{H}_{27} \mathrm{~N}_{3} \mathrm{O}_{3}$ & 537.61 & 78.19 & 5.06 & 7.82 & ----- \\
\hline & Benzene & 537.61 & & 78.22 & 5.10 & 7.85 & \\
\hline \multirow[t]{2}{*}{$14 i$} & 230 & $\mathrm{C}_{37} \mathrm{H}_{25} \mathrm{~N}_{3} \mathrm{O}_{2}$ & 543.19 & 81.75 & 4.64 & 7.73 & ----- \\
\hline & Benzene & 543.61 & & 81.80 & 4.68 & 7.70 & \\
\hline \multirow[t]{2}{*}{$14 j$} & 328 & $\mathrm{C}_{31} \mathrm{H}_{21} \mathrm{~N}_{3} \mathrm{O}_{2}$ & 467.16 & 79.64 & 4.53 & 8.99 & ----- \\
\hline & Benzene & 467.16 & & 79.66 & 4.55 & 9.11 & \\
\hline
\end{tabular}




\section{Biological Screening}

The activities of some of the prepared compounds against representative Gram positive and negative bacteria were tested by the disk diffusion method [1, 8, 35]. The results are listed in Table 5 . From the data it is clear that compounds $\mathbf{6 b}, \mathbf{1 4} \mathbf{i}$ possess high activity against both types of bacteria, while compound $\mathbf{6 c}$ displays low activity. Compounds $\mathbf{1}$ and $\mathbf{6 b}$ possess high activity, compounds $\mathbf{2}$, 3a-3d, 4a, 5a, 5d, 14g, 14i possess moderate activity and compounds $6 \mathbf{c}$ and $13 a$ possess less activity against Gram positive strains. As far as Gram negative microorganisms are concerned, compound 14i showed high activity, while compounds 1, 2, 3a, 3b, 3d, 4a, 5a, 5d, 6b, 13a and 14g all displayed moderate activity and $\mathbf{3 c}$ and $\mathbf{6 c}$ possess less activity against such microorganisms.

Table 5. Antibacterial activity of select compounds*

\begin{tabular}{|c|c|c|c|c|}
\hline \multirow{2}{*}{$\begin{array}{c}\text { Compound } \\
\text { No }\end{array}$} & \multicolumn{2}{|c|}{ Gram positive bacteria } & \multicolumn{2}{c|}{ Gram negative bacteria } \\
\cline { 2 - 5 } & Staph. aureus & Staph. epidermis & E. coli & Pr. vulgaris \\
\hline $\mathbf{1}$ & ++ & +++ & + & ++ \\
$\mathbf{2}$ & ++ & ++ & ++ & ++ \\
3a & + & +++ & ++ & ++ \\
$3 \mathbf{b}$ & ++ & +++ & ++ & ++ \\
$\mathbf{3 c}$ & ++ & ++ & + & + \\
$\mathbf{3 d}$ & ++ & ++ & ++ & + \\
$\mathbf{4 a}$ & ++ & ++ & ++ & + \\
$\mathbf{5 a}$ & ++ & ++ & ++ & + \\
$\mathbf{5 d}$ & ++ & ++ & +++ & ++ \\
$\mathbf{6 b}$ & ++ & ++++ & ++ & + \\
$\mathbf{6 c}$ & ++ & + & + & ++ \\
$\mathbf{1 3 a}$ & + & ++ & ++ & ++ \\
$\mathbf{1 4 g}$ & ++ & + & ++ & ++ \\
$\mathbf{1 4}$ & $+\mathbf{i}$ & ++ & ++ & ++ \\
\hline
\end{tabular}

* Solvent: DMF, [c] = $250 \mu \mathrm{g} \mathrm{mL}{ }^{-1}$. Ratings: + = less active (inhibition zone 1-5 $\mathrm{mm}$ ); $++=$ moderately active (inhibition zone $5-10 \mathrm{~mm}$ ), $+++=$ more active (inhibition zone 10-15 mm); ++++ = highly active (inhibition zone 15-20 mm); reference substance for Gram positive and Gram negative bacteria: ampicillin.

\section{Conclusions}

A novel synthesis of some new indolylpyridazinone derivatives by cyclocondensation of indolylbutyric acid $\mathbf{2}$ with hydrazine hydrate and its derivatives to give pyridazinone derivatives 3a-d is described. The reactions of pyridazinone 3a with $\mathrm{PCl}_{5} / \mathrm{POCl}_{3}$, arylsulphonyl chloride derivatives and aliphatic or aromatic aldehydes were studied, as were the behaviors of chloropyridazine derivatives towards hydrazine hydrate, carbohydrate hydrazones and aliphatic or aromatic amines. The structures of all new synthesized compounds were established from their spectral data and elemental analysis. Additionally, the antimicrobial activity of selected compounds against Gram positive and negative bacteria is reported. 


\section{Acknowledgements}

I thank Dr. Malka Mehsen in Girls College of Science, Dammam, Saudi Arabia for performing the biological tests

\section{Experimental}

\section{General}

Melting points were determined on Reichert hot stage microscope and are uncorrected. IR spectra were measured with a Nicolet Magna 520 instrument, using potassium bromide disks; results are given in $\mathrm{cm}^{-1}$. ${ }^{1} \mathrm{H}-\mathrm{NMR}$ and ${ }^{13} \mathrm{C}$-NMR spectra were recorded at 200 and $90.56 \mathrm{MHz}$, respectively, in DMSO$\mathrm{d}_{6}$ on a JEOL JNM-GX270 spectrometer. The chemical shifts are reported in parts per million (ppm) downfield from internal tetramethylsilane (TMS). Electron impact MS spectra were obtained on a JEOL JMS-HX 100 instrument at $70 \mathrm{eV}$. Elemental microanalysis was done on a Carlo Erba analyzer model 110. Suitable crystals were grown by slow crystallization from methanol, ethanol and benzene.

\section{4-Anthracen-9-yl-4-oxo-but-2-enoic acid (1)}

Compound 1 was prepared following the literature procedure [1, 20, 27]. It was obtained in 85\% yield as white crystals; IR $\left(\mathrm{cm}^{-1}\right)$ : $1608(\mathrm{C}=\mathrm{C}), 1662$ (ketone $\mathrm{C}=\mathrm{O}$ ), 1699 (acid $\mathrm{C}=\mathrm{O}$ ), 3054, 2970, 2927, 2869 (C-H), 4200-3400 (acid OH); ${ }^{1} \mathrm{H}-\mathrm{NMR}$ : 7.20-8.50 (m, 11H, Ar-H and vinyl), 11 (br, 1H, $\mathrm{COOH})$; ${ }^{13} \mathrm{C}-\mathrm{NMR}: 127-144$ (16C, Ar and vinyl), 170, 188.10 (2C, acid and ketone $\underline{\mathrm{C}}=\mathrm{O}$ ). Its physical properties, mass spectral data and elemental analysis are given in Table 1.

\section{4-Anthracen-9-yl-2-(1H-indol-3-yl)-4-oxo-butyric acid (2).}

Indole (10 mmol) was added to a solution of 4-anthracen-9-yl-4-oxo-but-2-enoic acid (1, $10 \mathrm{mmol})$ in dry benzene $(10 \mathrm{~mL})$ and the reaction mixture was refluxed for $6 \mathrm{hr}$. The solid that separated on cooling was recrystallized from benzene to give compound 2 as white crystals, yield $80 \%$; IR $\left(\mathrm{cm}^{-1}\right)$ : 1618 (C=C), 1674 (ketone C=O), 1708 (acid C=O), 3030, 3070, 2926, 2853 (C-H), 3414 (indole N-H); ${ }^{1} \mathrm{H}-\mathrm{NMR}$ : 3.40 (d, 2H, $\mathrm{CH}_{2}$ ), 4.04 (t, 1H, CH), 6.51 (s, 1H, indole $\left.\mathrm{CH}\right), 7.02-7.91(\mathrm{~m}, 13 \mathrm{H}, \mathrm{Ar}-\mathrm{H})$, 10.08 (br, 1H, NH), 11.10 (br, $1 \mathrm{H}, \mathrm{COOH}) ;{ }^{13} \mathrm{C}-\mathrm{NMR}$ : 40-41 (2C, $\underline{\mathrm{CH}}_{2}-\underline{\mathrm{CH}}$ ), 112.50-136.50 (21C, Ar), 123 (1C, indole $\underline{\mathrm{C}-\mathrm{NH}}$ ), 175.30, 198.90 (2C, acid and ketone $\underline{\mathrm{C}}=\mathrm{O}$ ). Physical properties, mass spectral data and elemental analysis for compound 2 are given in Table 1.

\section{General method for the preparation of 3a-d:}

Hydrazine hydrate derivatives $(10 \mathrm{mmol})$ were added to a solution of $\mathbf{2}(10 \mathrm{mmol})$ in dry benzene (5 mL) and the resulting reaction mixture was refluxed for $6 \mathrm{hr}$. The solid that separated on cooling was recystallized from benzene to give compounds 3a-d. Their physical properties, mass spectral data and elemental analysis are given in Table 1. 
6-Anthracen-9-yl-4-(1H-indol-3-yl)-4,5-dihydro-2H-pyridazin-3-one (3a).

Obtained from hydrazine hydrate and 2 as white needles, yield 81\%; IR ( $\left.\mathrm{cm}^{-1}\right): 1604(\mathrm{C}=\mathrm{C}), 1635$ $(\mathrm{C}=\mathrm{N}), 1671$ (pyridazinone $\mathrm{C}=\mathrm{O}), 3080,3010,3054,2957(\mathrm{C}-\mathrm{H}), 3100-3272(-\mathrm{OH}), 3411-3375$ (pyridazinone and indole N-H); ${ }^{1} \mathrm{H}-\mathrm{NMR}$ : $3.35\left(\mathrm{~d}, 2 \mathrm{H}, \mathrm{CH}_{2}\right), 4.36(\mathrm{t}, 1 \mathrm{H}, \mathrm{CH}), 6.81(\mathrm{~s}, 1 \mathrm{H}$, indole $\mathrm{CH}$ ), 6.90-7.91 (m, 13H, Ar-H), 10.9-12.40 (br, 2H, pyridazinone and indole $\mathrm{NH}$ ); ${ }^{13} \mathrm{C}-\mathrm{NMR}$ : 33.8040.10 (2C, $\left.\underline{\mathrm{CH}_{2}}-\underline{\mathrm{CH}}\right), 111.20-136$ (21C, Ar), 123 (1C, indole $\left.\underline{\mathrm{C}}-\mathrm{NH}\right), 153.34$ (1C, $\left.\underline{\mathrm{C}}=\mathrm{N}-\mathrm{N}\right), 170$ (1C, $\mathrm{C}=\mathrm{O})$.

6-Anthracen-9-yl-4-(1H-indol-3-yl)-2-phenyl-4,5-dihydro-2H-pyridazin-3-one (3b).

Obtained from phenyl hydrazine and 2 as white crystals, yield 79\%; IR ( $\left.\mathrm{cm}^{-1}\right)$ : 1605 (C=C), 1640 $(\mathrm{C}=\mathrm{N}), 1674$ (pyridazinone $\mathrm{C}=\mathrm{O}$ ), 3054, 3070, 2923, $2869(\mathrm{C}-\mathrm{H}), 3400-3411$ (indole $\mathrm{N}-\mathrm{H}$ ); ${ }^{1} \mathrm{H}-\mathrm{NMR}$ : 3.35 (d, 2H, $\mathrm{CH}_{2}$ ), 4.05 (t, 1H, CH), 6.80-7.82 (m, 19H, Ar-H), 11.02 (br, 1H, indole NH); ${ }^{13} \mathrm{C}-\mathrm{NMR}$ : 33.80-40.10 (2C, $\underline{\mathrm{CH}}_{2}-\underline{\mathrm{CH}}$ ), 111.20-136 (26C, Ar), 123 (1C, indole $\left.\underline{\mathrm{C}}-\mathrm{NH}\right), 141.20$ (1C, $\underline{\mathrm{C}}-\mathrm{N}-\mathrm{N}$ ), 153.34 (1C, $\underline{C}=\mathrm{N}-\mathrm{N}), 170$ (1C, pyridazinone $\underline{\mathrm{C}}=\mathrm{O})$.

3-Anthracen-9-yl-5-(1H-indol-3-yl)-6-oxo-5,6-dihydro-4H-pyridazine-1-carboxylic acid amide (3c).

Obtained from semicarbazide and 2 as a white solid, yield 75\%; IR ( $\left.\mathrm{cm}^{-1}\right)$ : 1605 (C=C), 1638 $(\mathrm{C}=\mathrm{N}$ ), 1650 (amide $\mathrm{C}=\mathrm{O}$ ), 1678 (pyridazinone $\mathrm{C}=\mathrm{O}$ ), 3070, 3054, 2926(C-H), 3320-3410 (indole and amide $\left.\mathrm{N}-\mathrm{H}, \mathrm{NH}_{2}\right)$; ${ }^{1} \mathrm{H}-\mathrm{NMR}$ : 3.34(d, $\left.2 \mathrm{H}, \mathrm{CH}_{2}\right)$, 4.05(t, $\left.1 \mathrm{H}, \mathrm{CH}\right), 4.50\left(\mathrm{br}, 2 \mathrm{H}, \mathrm{NH}_{2}\right), 6.81-7.80(\mathrm{~m}, 14 \mathrm{H}$,

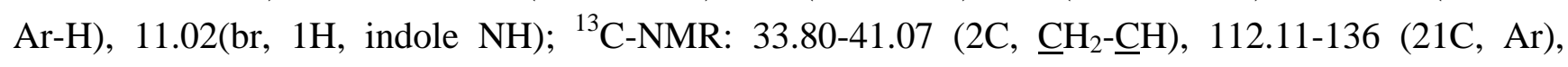
123.80 (1C, indole $\underline{\mathrm{C}}-\mathrm{NH}), 157.36(1 \mathrm{C}, \underline{\mathrm{C}}=\mathrm{N}-\mathrm{N}), 175,189$ (2C, pyridazinone and amide $\underline{\mathrm{C}}=\mathrm{O})$.

3-Anthracen-9-yl-5-(1H-indol-3-yl)-6-oxo-5,6-dihydro-4H-pyridazine-1-carbothioic acid amide (3d).

Obtained from thiosemicarbazide and 2 as white needles, yield 87\%; IR ( $\left.\mathrm{cm}^{-1}\right): 1270(\mathrm{C}=\mathrm{S}), 1604$ $(\mathrm{C}=\mathrm{C}), 1640(\mathrm{C}=\mathrm{N}), 1704$ (pyridazinone $\mathrm{C}=\mathrm{O}), 3057,3030,2959(\mathrm{C}-\mathrm{H}), 3350-3453$ (indole and carbothioic acid amide N-H, $\mathrm{NH}_{2}$ ); ${ }^{1} \mathrm{H}-\mathrm{NMR}$ : 3.34 (d, 2H, $\mathrm{CH}_{2}$ ), 4.05 (t, 1H, CH), 4.50 (br, 2H, $\mathrm{NH}_{2}$ ), 7.04-8.30 (m, 14H, Ar-H), 11.02 (br, 1H, indole NH); ${ }^{13} \mathrm{C}-\mathrm{NMR}$ : 33.80-41.07 (2C, $\left.\underline{\mathrm{CH}}_{2}-\underline{\mathrm{CH}}\right), 111.56-$ 133.41 (21C, Ar), 122.83 (1C, indole $\underline{\mathrm{C}}-\mathrm{NH}), 155$ (1C, $\underline{\mathrm{C}}=\mathrm{N}-\mathrm{N}), 177$ (1C, pyridazinone $\underline{\mathrm{C}}=\mathrm{O}$ ), 183 (1C, $\underline{C}=S)$.

3-(6-Anthracen-9-yl-3-chloropyridazin-4-yl)-1H-indole (4a).

$\mathrm{POCl}_{3}(5 \mathrm{~mL})$ was added to $3 \mathbf{a}(10 \mathrm{mmol})$ and the reaction mixture was heated on oil bath for 30 min, set aside to cool and then poured onto crushed ice $(60 \mathrm{~g})$, filtered, washed well with water and recrystallized from benzene to give 4a as a brown solid, yield 81\%; IR ( $\left.\mathrm{cm}^{-1}\right)$ : $756(\mathrm{C}-\mathrm{Cl}), 1605$ (C=C), 1640 (C=N), 3057, 3047, 2923, 2869 (C-H), 3391 (indole N-H); ${ }^{1} \mathrm{H}-\mathrm{NMR}:$ 7.30-8.30 (m, 15H, Ar-H), 10.90 (br, 1H, indole NH); ${ }^{13} \mathrm{C}-\mathrm{NMR}:$ 111.10-135 (23C, Ar), 123 (1C, indole $\left.\mathrm{C}-\mathrm{NH}\right), 152$ (1C, $\mathrm{N}-\mathrm{N}=\underline{\mathrm{C}}-\mathrm{Cl}), 160(1 \mathrm{C}, \underline{\mathrm{C}}=\mathrm{N}-\mathrm{N})$. Physical properties, mass spectral data and elemental analysis for compound $\mathbf{4 a}$ are given in Table 2. 
3-Anthracen-9-yl-6-chloropyridazine (4b).

Method A: $\mathrm{POCl}_{3}(5 \mathrm{~mL})$ was added to $3 a(10 \mathrm{mmol})$ and the reaction mixture was heated on oil bath for $3 \mathrm{hr}$, then set-aside poured on to $60 \mathrm{~g}$ crushed ice, filtered, washed well with water and recrystallized from benzene to give $\mathbf{4 b}$ as a brown solid, yield $81 \%$.

Method B: $\mathrm{POCl}_{3}(5 \mathrm{~mL})$ was added to $8(10 \mathrm{mmol})$ and the reaction mixture was heated on oil bath for $30 \mathrm{~min}$, then set-aside poured on to $60 \mathrm{~g}$ crushed ice, filtered, washed well with water and recrystallized from benzene to give $\mathbf{4 b}$ as a brown solid, yield 87\%; IR ( $\left.\mathrm{cm}^{-1}\right)$ : $752(\mathrm{C}-\mathrm{Cl}), 1605$ $(\mathrm{C}=\mathrm{C}), 1648$ (C=N), 3057, 3028 (C-H); ${ }^{1} \mathrm{H}-\mathrm{NMR}: 7.32-8.28$ (m, 11H, Ar-H); ${ }^{13} \mathrm{C}-\mathrm{NMR}: 111-132$ (16C, Ar), 151 (1C, N-N=C-Cl), $158(1 \mathrm{C}, \underline{\mathrm{C}}=\mathrm{N}-\mathrm{N})$. Physical properties, mass spectral data and elemental analysis for compound $\mathbf{4 b}$ are given in Table 2.

General procedure for the reaction of chloropyridazine 4a with carbohydrate hydrazones.

The appropriate carbohydrate hydrazone $(1 \mathrm{mmol})$ was added to a mixture of $\mathbf{4 a}(1 \mathrm{mmol})$ in ethanol $(5 \mathrm{~mL})$ and the reaction mixture was refluxed for $6 \mathrm{hr}$. The solid that separated on cooling was recystallized from ethanol to give compounds 5a-d. Physical properties, mass spectral data and elemental analysis for compounds 5a-d are given in Table 2.

(2R,3S,4S)-5-\{[6-Anthracen-9-yl-4-(1H-indol-3-yl)pyridazin-3-yl] hydrazono\}pentane-1,2,3,4,-tetraol (5a).

Ribose hydrazone gave compound 5a as an orange solid, yield 57\%; IR ( $\mathrm{cm}^{-1}$ ): 1605 (C=C), 1615 $(\mathrm{N}=\mathrm{N}), 1657$ (C=N), 3060, 2967, 2926, 2869 (C-H), 3430-3250 (indole and hydrazone N-H, NH$\mathrm{N}=\mathrm{C}), 3750-3250(\mathrm{O}-\mathrm{H})$; ${ }^{1} \mathrm{H}-\mathrm{NMR}$ : 3.32-3.50 (m, 3H, aliphatic $\left.\mathrm{CH}-\mathrm{OH}\right), 3.68$ (d, 2H, aliphatic $\mathrm{CH}_{2^{-}}$ $\mathrm{OH}$ ), 3.90 (s, 1H, NH-N), 4.85 (br, 4H, OH), 7- 8.27 (m, 15H, Ar-H), 7.53 (s, 1H, CH=N), 10.90 (br, $1 \mathrm{H}$, indole $\mathrm{NH}$ ); ${ }^{13} \mathrm{C}-\mathrm{NMR}$ : 60.9-74.60 (4C, aliphatic $\mathrm{C}-\mathrm{O}$ ), 111-138.70 (23C, Ar), 122.60 (1C, indole C-NH), 148-154 (2C, $\underline{C}=\mathrm{N}-\mathrm{N}), 159.20(1 \mathrm{C}, \mathrm{N}-\underline{\mathrm{C}}=\mathrm{N})$.

(2R,3R,4R,5S)-6-\{[6-Anthracen-9-yl-4-(1H-indol-3-yl)pyridazin-3-yl]-hydrazono\}-hexane-1,2,3,4,5pentaol (5b).

Glucose hydrazone gave compound $5 \mathbf{b}$ as a yellow solid, yield 52\%; IR ( $\left.\mathrm{cm}^{-1}\right): 1600$ (C=C), 1637 $(\mathrm{C}=\mathrm{N}), 3060,2960,2925$ (C-H), 3414-3197 (indole and hydrazone N-H), 3625-3620 (O-H); ${ }^{1} \mathrm{H}-\mathrm{NMR}$ : 3.30-3.44 (m, 4H, aliphatic $\mathrm{CH}-\mathrm{OH}), 3.68$ (m, 2H, aliphatic $\left.\mathrm{CH}_{2}-\mathrm{OH}\right), 3.90$ (s, $\left.1 \mathrm{H}, \mathrm{NH}-\mathrm{N}\right), 4.85$ (br, 5H, OH), 6.80-8.50 (m, 15H, Ar-H), 7.53 (s, 1H, CH=N), 10.90 (br, 1H, indole NH); ${ }^{13} \mathrm{C}-\mathrm{NMR}$ : 62.10-

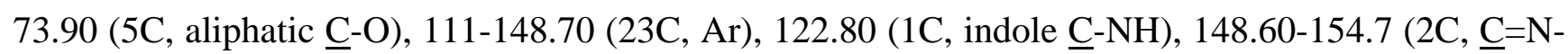
N), $164(1 \mathrm{C}, \mathrm{N}-\underline{\mathrm{C}}=\mathrm{N})$. 
(2R,3S,4R,5S)-6-\{[6-Anthracen-9-yl-4-(1H-indol-3-yl)pyridazin-3-yl]hydrazono\}-hexane-1,2,3,4,5pentaol (5c).

Galactose hydrazone gave compound 5c as a white solid, yield 55\%; IR $\left(\mathrm{cm}^{-1}\right)$ : 1565 (HN-N=C), $1605(\mathrm{C}=\mathrm{C}), 1640(\mathrm{C}=\mathrm{N}), 3057,2960,2923,2890(\mathrm{C}-\mathrm{H}), 3460-3412$ (indole and hydrazone $\mathrm{N}-\mathrm{H}$ ), 3629-3400 (O-H); ${ }^{1} \mathrm{H}-\mathrm{NMR}$ : 2.50-3.48 (m, 4H, aliphatic $\left.\mathrm{CH}-\mathrm{OH}\right), 3.60$ (m, 2H, aliphatic $\mathrm{CH}_{2}-\mathrm{OH}$ ), 3.91 (s, 1H, NH-N), 4.85 (br, 5H, OH), 7.00-8.10 (m, 15H, Ar-H), 7.51 (s, 1H, CH=N), 10.91 (br, 1H, indole $\mathrm{NH}$ ); ${ }^{13} \mathrm{C}-\mathrm{NMR}$ : 61.10-73.90 (5C, aliphatic $\left.\underline{\mathrm{C}}-\mathrm{O}\right), 111-148.70$ (23C, Ar), 122 (1C, indole $\underline{\mathrm{C}}$ NH), 148.60-154.70 (2C, $\underline{C}=\mathrm{N}-\mathrm{N}), 159.20$ (1C, N-C $=\mathrm{N})$.

6-\{[6-Anthracen-9-yl-4-(1H-indol-3-yl)pyridazin-3-yl] hydrazono\}-3-(3,4,5-trihydroxy-6-hydroxymethyltetrahydropyran-2-yloxy)-hexane-1,2,4,5-tetraol (5d).

Lactose hydrazone gave compound 5d as a white solid, yield 58\%; IR $\left(\mathrm{cm}^{-1}\right): 1570(\mathrm{HN}-\mathrm{N}=\mathrm{C})$, 1605 (C=C), 1638 (C=N), 3057, 2960, 2923(C-H), 3412-3412 (indole and hydrazone N-H), 3635-3390 (O-H); ${ }^{1} \mathrm{H}-\mathrm{NMR}:$ 2.99-3.76(m, 9H, aliphatic $\left.\mathrm{CH}-\mathrm{OH}\right), 3.66-3.68\left(\mathrm{~m}, 4 \mathrm{H}\right.$, aliphatic $\left.\mathrm{CH}_{2}-\mathrm{OH}\right), 3.90$ (s, 1H, NH-N), 4.85 (br, 8H, OH), 7.00-8.50 (m, 15H, Ar-H), 7.50 (s, 1H, CH=N), 10.90 (br, 1H, indole, $\mathrm{NH}$ ); ${ }^{13} \mathrm{C}-\mathrm{NMR}:$ 62.50-72.10 (10C, aliphatic $\underline{\mathrm{C}-\mathrm{O}),} 96.50$ (1C, O-C-O), 111-148.70 (23C, Ar), 122 (1C, indole $\underline{\mathrm{C}}-\mathrm{NH}), 148.60-154.70(2 \mathrm{C}, \underline{\mathrm{C}}=\mathrm{N}-\mathrm{N}), 159.20$ (1C, N-C $=\mathrm{N})$.

\section{General reaction of chloropyridazine $\mathbf{4 a}$ with aliphatic or aromatic amines}

The aliphatic or aromatic amine (1 mmol) was added to a mixture of $4 \mathbf{4 a}(1 \mathrm{mmol})$ in dry benzene ( $5 \mathrm{~mL}$ ) and the reaction mixture was heated in oil bath for $6 \mathrm{hr}$. The solid that separated on cooling was recrystallized from benzene to give compounds 6a-f. Their physical properties, mass spectral data and elemental analysis are given in Table 2.

[6-Anthracen-9-yl-4-(1H-indol-3-yl)-pyridazin-3-yl]-methylamine (6a).

Methylamine gave 6a as a white solid, yield 67\%; IR $\left(\mathrm{cm}^{-1}\right)$ : $1619(\mathrm{C}=\mathrm{C}), 1637(\mathrm{C}=\mathrm{N}), 3054$, 3030, 2957, 2923 (C-H), 3413-3406 (indole and secondary amine N-H); ${ }^{1} \mathrm{H}-\mathrm{NMR}$ : 0.83 (s, 3H, $\mathrm{CH}_{3}$ ), 3.89 (br, $1 \mathrm{H}$, secondary amine $\mathrm{NH}$ ), 7.05-7.90 (m, 15H, Ar-H), 11.12 (br, $1 \mathrm{H}$, indole $\mathrm{NH})$; ${ }^{13} \mathrm{C}-\mathrm{NMR}$ : 37.50 (1C, $\left.\mathrm{N}-\underline{\mathrm{CH}}_{3}\right), 111-136$ (23C, Ar), 123 (1C, indole $\left.\underline{\mathrm{C}}-\mathrm{NH}\right), 148.60$ (1C, N-ㄷ=ㄱ), 159.2 (1C, $\underline{\mathrm{C}}=\mathrm{N}-\mathrm{N})$.

[6-Anthracen-9-yl-4-(1H-indol-3-yl)-pyridazin-3-yl]-ethylamine (6b).

Ethylamine gave $\mathbf{6 b}$ as a brown solid, yield 52\%; IR $\left(\mathrm{cm}^{-1}\right)$ : $1617(\mathrm{C}=\mathrm{C}), 1635(\mathrm{C}=\mathrm{N}), 3054,2967$, 2925, 2869 (C-H), 3429-3410 (indole N-H and secondary amine N-H); ${ }^{1} \mathrm{H}-\mathrm{NMR}: 0.85$ (t, 3H, $\mathrm{CH}_{3}$ ), 1.60 (m, 2H, $\mathrm{CH}_{2}$ ), 3.89 (br, 1H, secondary amine $\mathrm{NH}$ ), 7.05-8.00 (m, 15H, Ar-H), 11.10 (br, 1H, indole $\mathrm{NH}$ ); ${ }^{13} \mathrm{C}-\mathrm{NMR}: 16.20$ (1C, $\underline{\mathrm{CH}}_{3}$ ), 44.20 (1C, N- $\mathrm{CH}_{2}$ ), 111-136 (23C, Ar), 123.41 (1C, indole C-NH), $151.10(1 \mathrm{C}, \underline{\mathrm{C}}=\mathrm{N}-\mathrm{N}), 160.10(1 \mathrm{C}, \mathrm{N}-\underline{\mathrm{C}}=\mathrm{N})$. 
[6-Anthracen-9-yl-4-(1H-indol-3-yl)-pyridazin-3-yl]-phenylamine (6c).

Aniline gave 6c as white needles, yield 72\%; IR $\left(\mathrm{cm}^{-1}\right)$ : $1617(\mathrm{C}=\mathrm{C}), 1638(\mathrm{C}=\mathrm{N}), 3057,2982$, 2973, 2869 (C-H), 3429-3400 (indole N-H and secondary amine N-H); ${ }^{1} \mathrm{H}-\mathrm{NMR}: 3.89$ (br, 1H, secondary amine $\mathrm{NH}$ ), 6.80-8.30 (m, 20H, Ar-H), 11.10 (br, $1 \mathrm{H}$, indole $\mathrm{NH}$ ); ${ }^{13} \mathrm{C}-\mathrm{NMR}$ : 111-148 (29C, Ar), 123 (1C, indole $\underline{C}-N H), 150.10(1 \mathrm{C}, \underline{\mathrm{C}}=\mathrm{N}-\mathrm{N}), 160.5$ (1C, N-C=N).

4-[6-Anthracen-9-yl-4-(1H-indol-3-yl)-pyridazin-3-ylamino] benzenesulfonic acid (6d).

Sulphanilic acid gave $\mathbf{6 d}$ as a gray solid, yield 51\%; IR $\left(\mathrm{cm}^{-1}\right)$ : 1160, $1423\left(\mathrm{SO}_{2}\right), 1601(\mathrm{C}=\mathrm{C})$, $1631(\mathrm{C}=\mathrm{N}), 3057,2960,2920(\mathrm{C}-\mathrm{H}), 3390$ (indole and secondary amine $\mathrm{N}-\mathrm{H}), 3200-3100(\mathrm{O}-\mathrm{H}) ;{ }^{1} \mathrm{H}-$ NMR: 3.89 (br, 1H, secondary amine NH), 6.00-8.50 (m, 19H, Ar-H), 11.10 (br, 1H, indole NH), 14.90 (br, $1 \mathrm{H}, \mathrm{SO}_{3} \mathrm{H}$ ); ${ }^{13} \mathrm{C}-\mathrm{NMR}$ : 111-148.70 (27C, Ar), 133.50 (1C, $\left.\underline{\mathrm{C}}-\mathrm{S}\right), 124$ (1C, indole $\underline{\mathrm{C}}-\mathrm{NH}$ ), $148.60(1 \mathrm{C}, \underline{\mathrm{C}}=\mathrm{N}-\mathrm{N}), 150.50$ (1C, $-\mathrm{NH}-\mathrm{Ph}), 159(1 \mathrm{C}, \mathrm{N}-\underline{\mathrm{C}}=\mathrm{N})$.

[6-Anthracen-9-yl-4-(1H-indol-3-yl)-pyridazin-3-yl]-naphthalen-1-yl amine (6e).

$\alpha$-Naphthylamine gave 6 e as a light brown solid, yield 58\%; IR $\left(\mathrm{cm}^{-1}\right)$ : $1616(\mathrm{C}=\mathrm{C}), 1640(\mathrm{C}=\mathrm{N})$, 3054, 2999, 2973 (C-H), 3429-3401 (indole and secondary amine N-H); ${ }^{1} \mathrm{H}-\mathrm{NMR}$ : 3.89 (br, 1H, secondary amine $\mathrm{NH}$ ), 6.70-8.27 (m, 22H, Ar-H), 11.10 (br, $1 \mathrm{H}$, indole $\mathrm{NH})$; ${ }^{13} \mathrm{C}-\mathrm{NMR}$ : 109.40141.60 (33C, Ar), 122 (1C, indole $\underline{\mathrm{C}}-\mathrm{NH}), 148.60$ (1C, $\underline{\mathrm{C}}=\mathrm{N}-\mathrm{N}), 161.20(1 \mathrm{C}, \mathrm{N}-\underline{\mathrm{C}}=\mathrm{N})$.

[6-Anthracen-9-yl-4-(1H-indol-3-yl)-pyridazin-3-yl] diphenylamine (6f).

Diphenylamine gave $6 \mathbf{f}$ as a dark brown solid, yield 51\%; IR: $1617(\mathrm{C}=\mathrm{C}), 1635(\mathrm{C}=\mathrm{N}), 3057$, 2982, 2973 (C-H), 3410 (indole N-H); ${ }^{1} \mathrm{H}-\mathrm{NMR}$ : 6.72-8.50 (m, 25H, Ar-H), 11.10 (br, 1H, indole $\mathrm{NH}$ ); ${ }^{13} \mathrm{C}-\mathrm{NMR}: 111-136$ (33C, Ar), 122.50 (1C, indole $\underline{\mathrm{C}-\mathrm{NH}}$ ), 142.90 (2C, N-C ), 150 (1C, $\underline{\mathrm{C}}=\mathrm{N}-\mathrm{N}$ ), $160(1 \mathrm{C}, \mathrm{N}-\underline{\mathrm{C}}=\mathrm{N})$.

2-Anthracen-9-yl-4-(1H-indol-3-yl)-1,9-a,10-triaza-anthracen-9-one (7).

Anthranilic acid (1 mmol) was added to a mixture of $\mathbf{4 a}(1 \mathrm{mmol})$ in dry benzene $(5 \mathrm{~mL})$ and the reaction mixture was refluxed for $6 \mathrm{hr}$. The solid that separated on cooling was recystallized from benzene to give 7 as a brown solid, yield 65\%; IR: $1600(\mathrm{C}=\mathrm{C}), 1638(\mathrm{C}=\mathrm{N}), 1665(\mathrm{C}=\mathrm{O}), 3050,2959$, 2925, 2869 (C-H), 3410-3290 (indole N-H); ${ }^{1} \mathrm{H}-\mathrm{NMR}$ : 7.10-8.60 (m, 19H, Ar-H), 12 (br, 1H, indole $\mathrm{NH})$; ${ }^{13} \mathrm{C}-\mathrm{NMR}:$ 111-147.70 (29C, Ar), 122 (1C, indole $\left.\underline{\mathrm{C}}-\mathrm{NH}\right), 155.50$ (1C, $\left.\underline{\mathrm{C}}=\mathrm{N}-\mathrm{N}\right), 160$ (1C, N$\underline{\mathrm{C}}=\mathrm{N}), 170(1 \mathrm{C}, \mathrm{N}-\underline{\mathrm{C}}=\mathrm{O})$.

6-Anthracen-9-yl-2H-pyridazin-3-one (8).

Hydrazine hydrate (1 mmol) was added to a solution of 4-anthracen-9-yl-4-oxo-but-2-enoic acid (1, $1 \mathrm{mmol})$ in dry benzene $(10 \mathrm{~mL})$ and the reaction mixture was refluxed for $6 \mathrm{hr}$. The solid that separated after concentration and cooling was recrystallized from benzene to give $\mathbf{8}$ as white needles, 
yield 85\%; IR ( $\left.\mathrm{cm}^{-1}\right)$ : $1604(\mathrm{C}=\mathrm{C}), 1630(\mathrm{C}=\mathrm{N}), 1670(\mathrm{C}=\mathrm{O}), 3070,3030,2957(\mathrm{C}-\mathrm{H}), 3411-3357$ (indole N-H); ${ }^{1} \mathrm{H}-\mathrm{NMR}$ : 6.50-8.60 (m, 11H, Ar-H), 10.20 (br, $1 \mathrm{H}$, pyridazinone $\mathrm{NH}$ ); ${ }^{13} \mathrm{C}-\mathrm{NMR}$ : $111-$ 135.70 (16C, Ar), 155 (1C, $\underline{C}=N-N), 168$ (1C, $\underline{C}=0)$.

6-Anthracen-9-yl-pyridazin-3-yl) hydrazine (9).

Hydrazine hydrate $(1 \mathrm{mmol})$ was added to a solution of $\mathbf{4 b}(1 \mathrm{mmol})$ in dry benzene $(10 \mathrm{~mL})$ and the reaction mixture was refluxed for hr. The solid that separated was recrystallized from dry benzene to give 9 as buff crystals, yield 70\%; IR $\left(\mathrm{cm}^{-1}\right)$ : $1605(\mathrm{C}=\mathrm{C}), 1181(\mathrm{C}=\mathrm{N}), 3054,3015,2958(\mathrm{C}-\mathrm{H})$, 3327-3139 (hydrazine $\mathrm{NHNH}_{2}$ ); ${ }^{1} \mathrm{H}-\mathrm{NMR}$ : 3.89-10 (br, 3H, NH-NH ), 6.59-8.30 (m, $\left.11 \mathrm{H}, \mathrm{Ar}-\mathrm{H}\right) ;{ }^{13} \mathrm{C}-$ NMR: 111-137 (16C, Ar), 158 (1C, $\underline{C}=\mathrm{N}-\mathrm{N}), 160$ (1C, N-C=NHNH${ }_{2}$ ).

\section{3-Anthracen-9-yl-6-(3,5-dimethylpyrazol-1-yl)pyridazine (10).}

Acetylacetone $(1 \mathrm{mmol})$ was added to a mixture of $9(1 \mathrm{mmol})$ in methanol $(10 \mathrm{~mL})$ and the reaction mixture was refluxed for $5 \mathrm{hr}$. The solid that separated after cooling was recrystallized from methanol to give 10 as yellow crystals, yield 62\%; IR $\left(\mathrm{cm}^{-1}\right)$ : $1604(\mathrm{C}=\mathrm{C}), 1635(\mathrm{C}=\mathrm{N}), 3050,2959$, 2925, 2869 (C-H); ${ }^{1} \mathrm{H}-\mathrm{NMR}: 2.93$ (s, 6H, 2CH ), 6.50-8.270 (m, 12H, Ar-H); ${ }^{13} \mathrm{C}-\mathrm{NMR}: 14.20$ (2C, 2CH$\left.{ }_{3}\right), 111-135$ (17C, Ar), $148(2 \mathrm{C}, \underline{\mathrm{C}}=\mathrm{N}), 155(1 \mathrm{C}, \underline{\mathrm{C}}=\mathrm{N}-\mathrm{N}), 160(1 \mathrm{C}, \mathrm{N}-\underline{\mathrm{C}}=\mathrm{N})$.

\section{6-Anthracen-9-yl-3-methylamino-pyridazine (11).}

Methylamine ( $1 \mathrm{mmol})$ was added to a mixture of $\mathbf{4} \mathbf{b}(1 \mathrm{mmol})$ and the reaction mixture was heated for $4 \mathrm{hr}$ on an oil-bath at $140{ }^{\circ} \mathrm{C}$; then cooled and triturated with methanol. The solid that separated was recrystallized from methanol to give $\mathbf{1 1}$ as white crystals, yield 56\%; IR (cm $\left.{ }^{-1}\right): 1604$ $(\mathrm{C}=\mathrm{C}), 1635$ (C=N), 3050, 2959, 2925, 2869 (C-H), 3412-3412 (indole and hydrazone N-H); ${ }^{1} \mathrm{H}-$ NMR: 2.50 (d, 3H, $\mathrm{CH}_{3}$ ), 6.30-8.32 (m, 11H, Ar-H), 4.20 (br, $1 \mathrm{H}$, amine $\left.\mathrm{NH}\right) ;{ }^{13} \mathrm{C}-\mathrm{NMR}$ : 35.30 (1C, $\left.\mathrm{CH}_{3}\right), 113-135$ (16C, Ar), 155.60 (1C, $\left.\underline{C}=\mathrm{N}-\mathrm{N}\right), 159$ (1C, N-C=N).

(6-Anthracen-9-yl-pyridazin-3-yl)trimethylammonium iodide (12).

Excess methyl iodide (5 mL) was added to a mixture of $11(1 \mathrm{mmol})$ in methanol $(10 \mathrm{~mL})$ and the reaction mixture was refluxed for $8 \mathrm{hr}$. After evaporation of all the solvent, the solid residue was recrystallized from methanol to give 12 as white crystals, yield 85\%; IR ( $\left.\mathrm{cm}^{-1}\right)$ : 1604 (C=C), 1635 (C=N), 3050, 2959, 2925, 2869 (C-H), 3412-3412 (N-N); ${ }^{1} \mathrm{H}-\mathrm{NMR}: 2.89$ (s, 9H, 3CH $)$, 7.39-8.30 (m, 11H, Ar-H); ${ }^{13} \mathrm{C}-\mathrm{NMR}: 52$ (3C, N-CH 3 ), 125.30-135.10 (16C, Ar), 148 (1C, $\left.\underline{C}=\mathrm{N}-\mathrm{N}\right), 159$ (1C, N$\underline{\mathrm{C}}=\mathrm{N})$.

6-Anthracen-9-yl-2-benzenesulfonyl-4-(1H-indol-3-yl)-4,5-dihydro-2H-pyridazin-3-one (13a).

Benzenesulfonyl chloride ( $1 \mathrm{mmol}$ ) was added to a mixture of $3 \mathbf{a}$ (1 mmol), anhydrous $\mathrm{K}_{2} \mathrm{CO}_{3}(1$ mmol) in dry acetone ( $5 \mathrm{~mL}$ ) and the reaction mixture was refluxed for $24 \mathrm{hr}$. The solid that separated on cooling was recystallized from benzene to give 13a as a white solid, yield 75\%; IR ( $\left.\mathrm{cm}^{-1}\right)$ : 1337 - 
$1175\left(\mathrm{SO}_{2}\right), 1616(\mathrm{C}=\mathrm{C}), 1637(\mathrm{C}=\mathrm{N}), 1670$ (pyridazinone $\mathrm{C}=\mathrm{O}$ ), 3054, 2957, 2923, 2869 (C-H), 3406 (indole N-H); ${ }^{1} \mathrm{H}-\mathrm{NMR}: 2.14$ (d, 2H, $\mathrm{CH}_{2}$ ), 3.50 (t, 1H, CH), 6.90-7.69 (m, 19H, Ar-H), 11.02 (br, 1H, indole $\mathrm{NH}$ ); ${ }^{13} \mathrm{C}-\mathrm{NMR}$ : 35.80 (1C, $\underline{\mathrm{CH}}_{2}$ ), 43.80 (1C, $\underline{\mathrm{CH}}$ ), 111-140.20 (27C, Ar), 123 (1C, indole $\underline{\mathrm{C}}-$ $\mathrm{NH}), 157$ (1C, $\underline{\mathrm{C}}=\mathrm{N}-\mathrm{N}), 176$ (1C, pyridazinone $\underline{\mathrm{C}}=\mathrm{O})$.

6-Anthracen-9-yl-4-(1H-indol-3-yl)-2-(toluene-4-sulfonyl)-4,5-dihydro-2H-pyridazin-3-one (13b).

4-Toluenesulfonyl chloride ( $1 \mathrm{mmol}$ ) was added to a mixture of $3 a$ ( $1 \mathrm{mmol})$, anhydrous $\mathrm{K}_{2} \mathrm{CO}_{3}(1$ $\mathrm{mmol}$ ) in dry acetone ( $5 \mathrm{~mL}$ ) and the reaction mixture was refluxed for $24 \mathrm{hr}$. The solid that separated on cooling was recystallized from benzene to give $\mathbf{1 3 b}$ as a white solid, yield 73\%; IR ( $\left.\mathrm{cm}^{-1}\right)$ : 1337 $1173\left(\mathrm{SO}_{2}\right), 1616(\mathrm{C}=\mathrm{C}), 1637(\mathrm{C}=\mathrm{N}), 1670$ (pyridazinone $\mathrm{C}=\mathrm{O}$ ), 3054, 2957, 2923, $2869(\mathrm{C}-\mathrm{H}), 3406$ (indole $\mathrm{N}-\mathrm{H}$ ); ${ }^{1} \mathrm{H}-\mathrm{NMR}: 2.10$ (s, 3H, $\mathrm{CH}_{3}$ ), 2.30 (t, $1 \mathrm{H}, \mathrm{CH}_{2}$ ), 3.70 (t, 1H, CH), 6.80-8.43 (m, 19H, Ar$\mathrm{H}), 10.02$ (br, $1 \mathrm{H}$, indole $\mathrm{NH}$ ); ${ }^{13} \mathrm{C}-\mathrm{NMR}$ : 20.90 (1C, $\left.\underline{\mathrm{CH}}{ }_{3}\right), 34.20$ (1C, $\underline{\mathrm{CH}}_{2}$ ), 43.90 (1C, $\left.\underline{\mathrm{CH}}-\mathrm{CO}\right)$, 111-142 (27C, Ar), 123 (1C, indole $\underline{\mathrm{C}}-\mathrm{NH}$ ), 157 (1C, $\underline{\mathrm{C}}=\mathrm{N}-\mathrm{N}), 178$ (1C, pyridazinone $\underline{\mathrm{C}}=\mathrm{O})$.

General reaction of pyridazinone 3a with some aliphatic or aromatic aldehydes

Aliphatic or aromatic aldehyde $(1 \mathrm{mmol})$ was added to a mixture of $3 a$ (1 mmol), $\mathrm{NaOH}$ (10\%) in ethanol $(5 \mathrm{~mL})$ and the reaction mixture was refluxed for $6 \mathrm{hr}$. The solid that separated on cooling was recystallized from benzene to give $\mathbf{1 4 a - j}$. Their physical properties, mass spectral data and elemental analysis are given in Table 4.

6-Anthracen-9-yl-4-(1H-indol-3-yl)5-methylene-4,5-dihydro-2H-pyridazin-3-one (14a).

Formaldehyde gave 14a as an orange solid, yield 53\%; IR $\left(\mathrm{cm}^{-1}\right)$ : $1615(\mathrm{C}=\mathrm{C}), 1637(\mathrm{C}=\mathrm{N}), 1671$ (pyridazinone C=O), 3054, 2957, 2925, 2869 (C-H), 3400-3413 (pyridazinone and indole N-H); ${ }^{1} \mathrm{H}-$ NMR: 3.36 (s, 2H, $\mathrm{CH}_{2}$ ), 4.40 (s, 1H, pyridazinone $\mathrm{CH}$ ), 7.04-8.50 (m, 14H, Ar-H), 11.02 (br, 2H, pyridazinone and indole $\mathrm{NH}) ;{ }^{13} \mathrm{C}-\mathrm{NMR}$ : 51 (1C, pyridazinone $\underline{\mathrm{CH}}$ ), 111-142 (23C, Ar and vinyl), 122 (1C, indole $\underline{\mathrm{C}}-\mathrm{NH}), 155(1 \mathrm{C}, \underline{\mathrm{C}}=\mathrm{N}-\mathrm{N}), 170$ (1C, pyridazinone $\underline{\mathrm{C}}=\mathrm{O})$.

6-Anthracen-9-yl-5-ethylidene-4-(1H-indol-3-yl)-4,5-dihydro-2H-pyridazin-3-one (14b).

Acetaldehyde gave 14b as an orange solid, yield 58\%; IR $\left(\mathrm{cm}^{-1}\right)$ : $1617(\mathrm{C}=\mathrm{C}), 1637(\mathrm{C}=\mathrm{N}), 1667$ (pyridazinone C=O), 3054, 2957, 2925, 2869 (C-H), 3400-3414 (pyridazinone and indole $\mathrm{N}-\mathrm{H}$ ); ${ }^{1} \mathrm{H}$ NMR: 1.70 (d, $3 \mathrm{H}, \mathrm{CH}_{3}$ ), 3.36 (m, 1H, vinyl $\mathrm{CH}$ ), 4.40 (s, $1 \mathrm{H}$, pyridazinone $\mathrm{CH}$ ), 6.67-8.22 (m, 14H, Ar-H), 11.02 (br, 2H, pyridazinone and indole $\mathrm{NH}$ ); ${ }^{13} \mathrm{C}-\mathrm{NMR}: 12-52.50$ (2C, pyridazinone $\underline{\mathrm{CH}}_{3}, \underline{\mathrm{CH}}$ ), 111-140 (23C, Ar and vinyl), 122.80 (1C, indole $\underline{\mathrm{C}}-\mathrm{NH}), 155.60$ (1C, $\underline{\mathrm{C}}=\mathrm{N}-\mathrm{N}), 170$ (1C, pyridazinone $\underline{\mathrm{C}}=\mathrm{O})$.

6-Anthracen-9-yl-5-benzylidene-4-(1H-indol-3-yl)-4,5-dihydro-2H-pyridazin-3-one (14c).

Benzaldehyde gave 14c as an orange solid, yield 62\%; IR $\left(\mathrm{cm}^{-1}\right)$ : $1616(\mathrm{C}=\mathrm{C}), 1637(\mathrm{C}=\mathrm{N}), 1671$ (pyridazinone C=O), 3054, 2957, 2925, 2869(C-H), 3400-3412 (pyridazinone and indole N-H); ${ }^{1} \mathrm{H}-$ 
NMR: 3.36 (s, 1H, vinyl CH), 4.40 (s, 1H, pyridazinone CH), 7.20-8.20 (m, 19H, Ar-H), 11.02 (br, $2 \mathrm{H}$, pyridazinone and indole $\mathrm{NH}$ ); ${ }^{13} \mathrm{C}-\mathrm{NMR}$ : 52.40 (1C, pyridazinone $\underline{\mathrm{CH}}$ ), 111-137 (29C, Ar and vinyl), 122 (1C, indole $\underline{\mathrm{C}}-\mathrm{NH}$ ), 155.60 (1C, $\underline{\mathrm{C}}=\mathrm{N}-\mathrm{N}), 168$ (1C, pyridazinone $\underline{\mathrm{C}}=\mathrm{O})$.

6-Anthracen-9-yl-5-(2-hydroxybenzylidene)-4-(1H-indol-3-yl)-4,5-dihydro-2H-pyridazin-3-one (14d).

2-Hydroxybenzaldehyde gave 14d as a brown solid, yield 60\%; IR ( $\left.\mathrm{cm}^{-1}\right)$ : 1619 (C=C), 1637 $(\mathrm{C}=\mathrm{N}), 1681$ (pyridazinone $\mathrm{C}=\mathrm{O}), 3059,2959,2927,2870(\mathrm{C}-\mathrm{H}), 3250-3500(\mathrm{O}-\mathrm{H}), 3400-3411$ (pyridazinone and indole N-H); ${ }^{1} \mathrm{H}-\mathrm{NMR}$ : 3.37 (s, $1 \mathrm{H}$, vinyl $\mathrm{CH}$ ), 4.40 (s, 1H, pyridazinone $\mathrm{CH}$ ), 6.628.52 (m, 18H, Ar-H), 5.50 (br, 1H, OH), 11.01 (br, 2H, pyridazinone and indole $\mathrm{NH}$ ); ${ }^{13} \mathrm{C}-\mathrm{NMR}: 52$ (1C, pyridazinone $\underline{\mathrm{CH}}$ ), 111-136.10 (28C, Ar and vinyl), 122.80 (1C, indole $\mathrm{C}-\mathrm{NH}), 155.60$ (1C, $\underline{\mathrm{C}}=\mathrm{N}$ N), 156(1C, $-\underline{C}-\mathrm{OH}), 168$ (1C, pyridazinone $\underline{\mathrm{C}}=\mathrm{O}$ ).

6-Anthracen-9-yl-5-(4-hydroxybenzylidene)-4-(1H-indol-3-yl)-4,5-dihydro-2H-pyridazin-3-one (14e).

4-Hydroxybenzaldehyde gave 14e as a buff solid, yield 72\%; IR $\left(\mathrm{cm}^{-1}\right)$ : $1595(\mathrm{C}=\mathrm{C}), 1640(\mathrm{C}=\mathrm{N})$, 1674 (pyridazinone $\mathrm{C}=\mathrm{O}$ ), 3054, 2957, 2925, 2869 (C-H), 3250-3500 (O-H), 3400-3414 (pyridazinone and indole $\mathrm{N}-\mathrm{H}$ ); ${ }^{1} \mathrm{H}-\mathrm{NMR}$ : 3.37 (s, $1 \mathrm{H}$, vinyl $\mathrm{CH}$ ), 4.40 (s, $1 \mathrm{H}$, pyridazinone $\mathrm{CH}$ ), 5.67 (br, $1 \mathrm{H}, \mathrm{OH}$ ), 6.44-8.17 (m, 18H, Ar-H), 11.02 (br, 2H, pyridazinone and indole $\mathrm{NH}$ ); ${ }^{13} \mathrm{C}-\mathrm{NMR}$ : 53 (1C, pyridazinone $\underline{\mathrm{C}} \mathrm{H}), 111-136$ (28C, Ar and vinyl), 122.80 (1C, indole $\underline{\mathrm{C}}-\mathrm{NH}), 155.60$ (1C, $\underline{\mathrm{C}}=\mathrm{N}-\mathrm{N})$, 157.50 (1C, $\underline{\mathrm{C}}-\mathrm{OH}), 168$ (1C, pyridazinone $\underline{\mathrm{C}}=\mathrm{O}$ ).

6-Anthracen-9-yl-4-(1H-indol-3-yl)-5-(2-methoxybenzylidene)-4,5-dihydro-2H-pyridazin-3-one (14f).

2-Methoxybenzaldehyde gave $\mathbf{1 4 f}$ as a brown solid, yield 50\%; IR ( $\left.\mathrm{cm}^{-1}\right)$ : 1590 (C=C), 1641 $(\mathrm{C}=\mathrm{N}), 1671$ (pyridazinone $\mathrm{C}=\mathrm{O}$ ), 3059, 2958, 2935, $2835(\mathrm{C}-\mathrm{H}), 3410-3424$ (pyridazinone and indole $\mathrm{N}-\mathrm{H}$ ); ${ }^{1} \mathrm{H}-\mathrm{NMR}$ : 3.36 (s, $1 \mathrm{H}$, vinyl $\left.\mathrm{CH}\right), 3.70\left(\mathrm{~s}, 3 \mathrm{H},-\mathrm{OCH}_{3}\right), 4.40$ (s, $1 \mathrm{H}$, pyridazinone $\mathrm{CH}$ ), 6.58-8.28 (m, 18H, Ar-H), 11.02 (br, 2H, pyridazinone and indole $\mathrm{NH}$ ); ${ }^{13} \mathrm{C}-\mathrm{NMR}$ : 52.40 (1C, pyridazinone $\underline{\mathrm{CH}}$ ), 56 (1C, O- $\underline{\mathrm{CH}}_{3}$ ), 111-138 (28C, Ar and vinyl), 122.80 (1C, indole $\underline{\mathrm{C}-\mathrm{NH}}$ ), 155.60

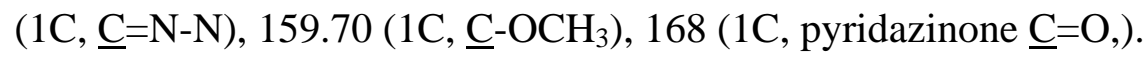

6-Anthracen-9-yl-4-(1H-indol-3-yl)-5-(4-methoxybenzylidene)-4,5-dihydro-2H-pyridazin-3-one (14g).

4-Methoxybenzaldehyde gave $\mathbf{1 4 g}$ as a brown solid, yield 72\%; IR ( $\left.\mathrm{cm}^{-1}\right)$ : 1613 (C=C), 1637 $(\mathrm{C}=\mathrm{N}), 1669$ (pyridazinone $\mathrm{C}=\mathrm{O}$ ), 3054, 2957, 2925, 2869 (C-H), 3400-3412 (pyridazinone and indole $\mathrm{N}-\mathrm{H}$ ); ${ }^{1} \mathrm{H}-\mathrm{NMR}$ : 3.37 (s, $1 \mathrm{H}$, vinyl $\mathrm{CH}$ ), 3.74 (s, 3H, $\mathrm{OCH}_{3}$ ), 4.37 (s, $1 \mathrm{H}$, pyridazinone $\mathrm{CH}$ ), 6.86-7.80 (m, 18H, Ar-H), 10.02, 11.02 (br, 2H, pyridazinone and indole $\mathrm{NH}) ;{ }^{13} \mathrm{C}-\mathrm{NMR}: 52$ (1C, pyridazinone $\underline{\mathrm{CH}}$,), 56 (1C, $\left.\mathrm{OCH}_{3}\right), 111-136$ (28C, Ar and vinyl), 122.80 (1C, indole $\left.\underline{\mathrm{C}}-\mathrm{NH}\right), 155.60$ (1C, $\left.\underline{\mathrm{C}}=\mathrm{N}-\mathrm{N}\right)$, 161 (1C, $\left.\underline{\mathrm{C}}-\mathrm{OCH}_{3}\right), 168$ (1C, pyridazinone $\underline{\mathrm{C}}=\mathrm{O}$,). 
6-Anthracen-9-yl-5-(2,4-dimethoxybenzylidene)-4-(1H-indol-3-yl-4,5-dihydro-2H-pyridazin-3-one (14h).

2,4-Dimethoxybenzaldehyde gave 14h as an orange solid, yield 51\%; IR ( $\left.\mathrm{cm}^{-1}\right)$ : 1601 (C=C), 1636 $(\mathrm{C}=\mathrm{N}), 1669$ (pyridazinone $\mathrm{C}=\mathrm{O}$ ), 3059, 2958, 2923, $2855(\mathrm{C}-\mathrm{H}), 3410-3424$ (pyridazinone and indole $\mathrm{N}-\mathrm{H}$ ); ${ }^{1} \mathrm{H}-\mathrm{NMR}$ : 3.36 (s, 1H, CH), 3.75 (s, 6H, $-\mathrm{OCH}_{3}$ ), 4.40 (s, 1H, pyridazinone $\mathrm{CH}$ ), 6.48-7.98 (m, 17H, Ar-H), 10.52 (br, 2H, pyridazinone and indole $\mathrm{NH}$ ); ${ }^{13} \mathrm{C}-\mathrm{NMR}: 52$ (1C, pyridazinone $\underline{\mathrm{CH}}$ ), 56.27 (2C, O- $\underline{\mathrm{CH}}_{3}$ ), 98-151 (27C, Ar and vinyl), 122 (1C, indole $\underline{\mathrm{C}}-\mathrm{NH}$ ), 155.60 (1C, $\left.\underline{\mathrm{C}}=\mathrm{N}-\mathrm{N}\right), 160-162$ (2C,

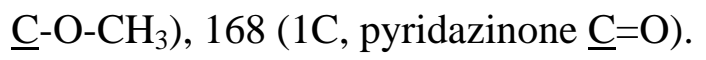

6-Anthracen-9-yl-5-(2-hydroxynaphthalen-1-yl-methylene)-4-(1H-indol-3-yl)-4,5-dihydro-2Hpyridazin-3-one (14i).

2-Hydroxynaphthaldehyde gave 14i as a brown solid, yield 58\%; IR ( $\left.\mathrm{cm}^{-1}\right)$ : 1619 (C=C), 1637 $(\mathrm{C}=\mathrm{N}), 1681$ (pyridazinone $\mathrm{C}=\mathrm{O}), 3059,2959,2927,2870(\mathrm{C}-\mathrm{H}), 3250-3500(\mathrm{O}-\mathrm{H}), 3400-3411$ (pyridazinone and indole $\mathrm{N}-\mathrm{H}$ ); ${ }^{1} \mathrm{H}-\mathrm{NMR}$ : 3.85 (s, $1 \mathrm{H}$, vinyl $\left.\mathrm{CH}\right), 4.40(\mathrm{~s}, 1 \mathrm{H}$, pyridazinone $\mathrm{CH}$ ), 6.47-7.95 (m, 20H, Ar-H), 5.62 (br, 1H, OH), 10.21 (br, $2 \mathrm{H}$, pyridazinone and indole $\mathrm{NH}$ ); ${ }^{13} \mathrm{C}-\mathrm{NMR}$ : 52.40 (1C, pyridazinone $\underline{\mathrm{CH}}$ ), 111-137 (32C, Ar and vinyl), 122.80 (1C, indole $\underline{\mathrm{C}}-\mathrm{NH}), 155.60$ (1C, $\underline{\mathrm{C}}=\mathrm{N}-\mathrm{N}), 156$ (1C, $\underline{-\mathrm{C}}-\mathrm{OH}), 170$ (1C, pyridazinone $\underline{\mathrm{C}}=\mathrm{O})$.

6-Anthracen-9-yl-5-furan-2-yl-methylene-4-(1H-indol-3-yl)-4,5-dihydro-2H-pyridazin-3-one (14j).

Furfuraldehyde gave 14j as a brown solid, yield 59\%; IR $\left(\mathrm{cm}^{-1}\right)$ : $1616(\mathrm{C}=\mathrm{C}), 1637(\mathrm{C}=\mathrm{N}), 1670$ (pyridazinone $\mathrm{C}=\mathrm{O}$ ), 3054, 2957, 2925, 2869 (C-H), 3400-3412 (pyridazinone and indole N-H), 1200 (C-O-C); ${ }^{1} \mathrm{H}$ NMR: 3.39 (s, 1H, vinyl CH), 4.37 (s, 1H, pyridazinone $\mathrm{CH}$ ), 6.30-8.55 (m, 17H, Ar-H), 11.02 (br, 2H, pyridazinone and indole $\mathrm{NH}$ ); ${ }^{13} \mathrm{C}-\mathrm{NMR}$ : 51 (1C, pyridazinone $\underline{\mathrm{CH}}$, ), 111-136 (25C, Ar and vinyl), 122.80 (1C, indole $\underline{\mathrm{C}}-\mathrm{NH}), 145-155.3$ (2C, furan $\mathrm{O}-\underline{\mathrm{C}}), 155.60$ (1C, $\underline{\mathrm{C}}=\mathrm{N}-\mathrm{N}), 168$ (1C, pyridazinone $\underline{\mathrm{C}}=\mathrm{O})$.

\section{References}

1. Sayed, G.H.; Sayed, M.A.; Mahmoud, M.R.; Shaaban, S.S. Synthesis and Reactions of New Pyidazinone Derivatives of Expected Antimicrobial Activities. Egypt. J. Chem. 2002, 45, 767776.

2. Katrusiak, A.; Katrusiak, A.; Baloniak, S. Reactivity of 6-Chloro-4- and 5-Hydrazino-2- Phenyl3(2H)-Pyridazinones with Vilsmeier Reagent. Tetrahedron 1994, 50, 12933-12940.

3. Okcelik, B.; Unlu, S.; Banoglu, E.; Kupeli, E.; Yesilada, E.; Sahin, M.F. Investigation of New Pyridazinone Derivatives for the Synthesis of Potent Analgesic and Anti-Inflammatory Compounds with Cyclooxygenase Inhibitory Activity. Arch. Pharm. Pharm. Med. Chem. 2003, 336, 406-412.

4. Dogruer, D.S.; Sahin, M.F.; Kupeli, E.; Yesilada, E. Synthesis and Analgesic and AntiInflammatory Activity of New Pyridazinones. Turk. J. Chem. 2003, 27, 727-738. 
5. Frolov, E.B.; Lakner, F.J.; Khvat, A.V.; Ivachtchenko, A.V. An Efficient Synthesis of Novel 1,3oxazolo [4,5-d] Pyridazinones. Tetrahedron Lett. 2004, 45, 4693-4696.

6. Banoglu, E.; Akoglu, C.; Unlu, S.; Kupeli, E.; Yesilada, E.; Sahin, M.F. Amide Derivatives of [6(5-Methyl-3-phenylpyrazole-1-yl)-3(2H)-Pyridazinone-2-yl]acetic Acids as Potential Analgesic anti-Inflammatory Compounds. Arch. Pharm. Pharm. Med. Chem. 2004, 337, 7-14.

7. Gokce, M.; Dogruer, D.; Sahin, F. Synthesis and Antinociceptive Activity of 6-Substituted-3pyridazinone Derivatives. Il Farmaco 2001, 56, 233-237.

8. Cao, S.; Qian, X.; Song, G.; Chai, B.; Jiang, Z. Synthesis and Antifeedant Activity of New Oxadiazolyl 3(2H)-pyridazinones. J. Agric. Food Chem. 2003, 51, 152-155.

9. Piaz, V.D.; Ciciani, G.; Giovannoni, M.P. 5-Acetyl-2-Methyl-4-Nitro-6-Phenyl-3(2H)Pyriazinone: Versatile Precursor to Hetero-Condensed Pyridazinones. Synthesis 1994, 669-671.

10. Ogretir, C.; Yarligan, S.; Demirayak, S. Spectroscopic Determination of Acid Dissociation Constants of Some Biologically Active 6-Phenyl-4,5-Dihydro-3(2H)-Pyridazinone Derivatives. $J$. Chem. Eng. Data 2002, 47, 1396-1400.

11. Barbaro, R.; Betti, L.; Botta, M.; Corelli,F.; Giannaccini, G.; Maccari, L.; Manetti, F.; Strappaghetti, G.; Corsano, S. Synthesis Biological Evaluation and Pharmacophore Generation of New Pyridazinone Derivatives with Affinity Toward $\alpha_{1}$ - and $\alpha_{2}$ - Adrenoceptors. J. Med. Chem. 2001, 44, 2118-2132.

12. Sircar, I. Synthesis of New 1,2,4-Triazolo[4,3-b]pyridazines and Related Compound. J. Heterocyclic Chem. 1985, 22, 1045-1048.

13. Coelho, A.; Sotelo, E.; Fraiz, N.; Yanez, M.; Laguna, R.; Cano, E.; Ravina, E. Pyridazines. Part 36: Synthesis and Antiplatelet Activity of 5-Substituted-6-Phenyl-3(2H)- Pyridazinones. Bioorg. Med. Chem. Lett. 2004, 14, 321-324

14. Sotelo, E.; Centeno, N.B.; Rodrigo, J.; Ravina, E. Pyridazine Derivatives. Part 27: A joint Theoretical and Experimental approach to the Synthesisi of 6-Phenyl-4,5-disubstituted3(2H)Pyridazinones. Tetrahedron Lett. 2002, 58, 2389-2395.

15. Sotelo, E.; Fraiz, N.; Yanez, M.; Terrades, V.; Laguna, R.; Cano, E.; Ravina, E. Pyridazines. Part XXIX: Synthesis and Platelet Aggregation Inhibition Activity of 5-Substituted-6-Phenyl-3(2H)Pyridazinones Novel Aspects of their Biological Action. Bioorg. Med. Chem. 2002, 10, 28732882.

16. Malinka, W.; Redzicka, A.; Lozach, O. New Derivatives of Pyrrolo[3,4-d]Pyridazinone and their Anticancer Effects. Il Farmaco 2004, 59, 457-462.

17. Youssef, A.S.; Marzouk, M.I.; Madkour, H.M.F.; El-Soll, A.M.A.; El-Hashash, M.A. Synthesis of some heterocyclic systems of anticipated biological activities via 6-aryl-4-pyrazol-1-yl-pyridazin3-one. Can. J. Chem. 2005, 83, 251-259.

18. Sotelo, E.; Pita, B.; Ravina, E. Pyridazines. Part 22: ${ }^{1}$ Highly Efficient Synthesis of Pharmacologically Useful 4-Cyano-6-Phenyl-5-Substituted-3(2H)-Pyridazinones. Tetrahedron Lett. 2000, 41, 2863-2866.

19. Sotelo, E.; Coelho, A.; Ravina, E. Pyridazine Derivatives 321): Stille-Based Approaches in the Synthesis of 5-Substituted-6-Phenyl-3(2H)-Pyridazinones. Chem. Pharm. Bull. 2003, 51, 427-430.

20. Kassab, R.R. Simple Synthesis and Reactions of Some New Pyridazinono Derivatives and their Antimicrobial Activity. Egypt. J. Chem. 2002, 45, 1055-1073. 
21. Toshiki, S.; Hiroshi, Y. Jpn. Kokai Tokkyo Koho JP 01,100,157, 1989; [Chem Abstr. 1989, 111, $153626 \mathrm{u}]$.

22. Masanori, S.; Toshiharu, O.; Hiroko, T. Jpn. Kokai Tokkyo Koho JP 01,31,763, 1989; [Chem Abstr. 1989, 111, 15623 r].

23. Tsutomu, F.; Yoichiro, N.; Tomokayu, G.; Amid Kazuimasa, Y. Jpn. Kokai Tokkyo Koho JP 61,148,160, 1987; [Chem Abstr. 1987, 106, 50037 v].

24. John, B.E.; James C.N.; Bayer, A.C. Eur. Pat. Appl. EP 409,027, 1991; [Chem. Abstr. 1991, 114, 228729 c].

25. Kamat, G.A.; Joshi, G.R.; Gadaginamath, S.G. Proc. Indian Acad. Sci. Chem. Sci. 1993, 105, 189.

26. Halasz, B.D.; Monsieurs, K.; Elias, O.; Karolyhazy, L.; Tapolcsanyi, P.; Maes, B.U.; Riedl, Z.; Hajos, G.; Dommisse, R.A.; Lemiere, G.L.; Kosmrlj, J.; Matyus, P. Synthesis of $5 H$ Pyridazino[4,5- $b$ ]Indoles and their benzofurane analogues utilizing an intramolecular Heck-Type Reaction. Tetrahedron. 2004, 60, 2283-2291.

27. Sayed, G.H.; El-Kady, M.Y.; Abd Elhalim, M.S. Synthesis and Reactions of Some $\alpha$-aryl-(4bromobenzoyl)propionic acids. Indian J. Chem. 1981, 20, 845-848.

28. Sayed, G.H.; Hamed, A.A.; Meligi, G.A.; Boraie, W.E.; Shafik, M. The Use of 4-(3,4Dichlorophenyl)-4-Oxo-2-(4-Antipyrinyl)-Butonoic Acid in the Preparation of Some New Heterocyclic Compounds With Expected Biological Activity. Molecules 2003, 8, 322-332.

29. Toth, G.; Molnar, S.; Tamas, T.; Borbely, I. An Efficient Synthesis of 4,5-Dihydro-3(2H)Pyridazinone Derivative. Synth. Commun. 1997, 27, 3513-3524.

30. Sayed, G.H.; Sayed, M.A.; Shaaban, S.S.; Mahmoud, M.R. Synthesis and Reactions of 4-(pBromophenyl)-4-Oxo-2-(4-Antipyrinyl)Butanoic Acid and Some Unexpected Products. Egypt. J. Chem. 2000, 43, 17-29.

31. Coates, W.J.; McKillop, A. One Pot Preparation of 6-Substituted 3(2H)-Pyridazinones from Ketones. Synthesis 1993, 334-342.

32. Kassab, R.R.; Sayed, G.H.; Radwan, A.M.; Abd El-Azzez, N. Some reactions with (biphenyl)-4(5-oxo-1,3-diphenyl-2-pyrazolin-4-yl)-4,5-dihydropyridazin-3-(2H)ones. Rev. Roum. Chim. 2001, 46, 649-655.

33. Jaihne, H.; Sayed, A.; Zaher, H.A.; Sherif, O. Reaction of 3-Chloro- and 3-Hydrazino-6-(ptolyl)pyridazines. Indian J. Chem. 1977, 250-251.

34. El-Hashash, M.A.; Amine, M.S.; Soliman, F.M.; Morsi, M.A. Behavior of aroylacyclic acids toward hydrazine hydrate and some on the cyclized products. J. Serb. Chem. Soc. 1992, 57, 563569.

35. Chung, K.T.; Chen, S.C.; Wong, T.Y.; Wei, C.I. Effects of Benzidine and Benzidine Analogues on Growth of Bacteria Including Azotobacter Vinelandii. Environ. Toxicol. Chem. 1998, 17, 271275.

Sample Availability: Available from the author.

(c) 2007 by MDPI (http://www.mdpi.org). Reproduction is permitted for noncommercial purposes. 